\title{
Combining Hybrid and One-Step-Ahead Smoothing for Efficient Short-Range Storm Surge Forecasting with an Ensemble Kalman Filter
}

\author{
NAILA F. RABOUdi AND BOUJEMAA AIT-EL-FQUIH \\ King Abdullah University of Science and Technology, Thuwal, Saudi Arabia \\ CLINT DAWSON \\ The University of Texas at Austin, Austin, Texas \\ IBRAHIM HOTEIT \\ King Abdullah University of Science and Technology, Thuwal, Saudi Arabia
}

(Manuscript received 27 November 2018, in final form 6 June 2019)

\begin{abstract}
This work combines two auxiliary techniques, namely the one-step-ahead (OSA) smoothing and the hybrid formulation, to boost the forecasting skills of a storm surge ensemble Kalman filter (EnKF) forecasting system. Bayesian filtering with OSA-smoothing enhances the robustness of the ensemble background statistics by exploiting the data twice: first to constrain the sampling of the forecast ensemble with the future observation, and then to update the resulting ensemble. This is expected to improve the behavior of EnKFlike schemes during the strongly nonlinear surges periods, but requires integrating the ensemble with the forecast model twice, which could be computationally demanding. The hybrid flow-dependent/static formulation of the EnKF background error covariance is then considered to enable the implementation of the filter with a small flow-dependent ensemble size, and thus less model runs. These two methods are combined within an ensemble transform Kalman filter (ETKF). The resulting hybrid ETKF with OSA smoothing is tested, based on twin experiments, using a realistic setting of the Advanced Circulation (ADCIRC) model configured for storm surge forecasting in the Gulf of Mexico and assimilating pseudo-observations of sea surface levels from a network of buoys. The results of our numerical experiments suggest that the proposed filtering system significantly enhances ADCIRC forecasting skills compared to the standard ETKF without increasing the computational cost.
\end{abstract}

\section{Introduction}

Storm surge, which occurs when sea level rises dramatically during a storm, is considered to be a catastrophic natural disaster and is, by far, the greatest threat to life and property along coastal regions. The storm that took place in the North Sea and flooded nearly $600 \mathrm{~km}^{2}$ of land in the United Kingdom and the Netherlands in February 1953 (McRobie et al. 2005), the surge associated with the Bhola cyclone that ravaged Bangladesh in November 1970 (Murty et al. 1986), and more recently, Hurricane Katrina that made landfall in New Orleans in August 2005 (Blake et al. 2007), were all responsible for thousands of deaths. The increase in the number of

\footnotetext{
Corresponding author: Ibrahim Hoteit, Ibrahim.hoteit@kaust. edu.sa
}

devastating storm surge events over the past few decades have raised the need for real-time forecasting systems in order to mitigate their effects (Butler et al. 2012). Providing timely accurate surge forecasts is crucial for the authorities in charge of evacuation and rescue plans to support decision-making and improve the management of public safety.

Coastal ocean models provide efficient numerical tools to predict sea level surges (Mel and Lionello 2014a,b). However, despite the continuous progress in computing resources that enabled their implementation on high-resolution grids, present-day models remain subject to significant sources of uncertainties (Butler et al. 2012; Siripatana et al. 2017). These include imperfect wind forcing, poorly known model parameters, incomplete physical properties, and uncertain boundary and initial conditions (Altaf et al. 2014). Computational storm 
surge models are further particularly sensitive to the input data and thus, any source of uncertainty might severely drift the model simulations and deteriorate its forecasting skills. Understanding and describing these uncertainties is crucial for many practical applications (e.g., the field of risk analysis and decision-making). Storm surge model forecasts can indeed be probabilistically unreliable when these sources of uncertainty are not incorporated adequately and therefore the need to integrate model predictions with an estimate of uncertainty has been pointed out by many authors (Beven 1989; McMillan and Brasington 2008; Zhong et al. 2010). This is motivated by the fact that such systems need to deliver, not only an accurate prediction of water levels, but also information about the uncertainty on the forecast and the probability to cross critical thresholds. Such forecasts can be produced using the ensemble prediction system (EPS) technique (Heaps 1983; Buizza and Palmer 1995; Buizza et al. 1999). Based on the chaos theory describing systems' behavior that are highly sensitive to the initial conditions, the method assesses uncertainty in forecasts by considering a set of different forecasts based on a set of different initial conditions, instead of a single "deterministic" forecast (Mel and Lionello 2014a,b, 2016). These initial conditions are designed to include the perturbations that amplify rapidly in time and are often formulated based on the singular vector technique (Buizza and Palmer 1995). Following the first attempt of Flowerdew et al. (2010) to describe an application of the EPS in operational surge prediction system, several EPS storm surge forecasting systems have become operational in several centers (e.g., Flowerdew et al. 2013; Mel and Lionello 2014a). Nowadays, data assimilation is recognized as the most efficient approach to mitigate the impact of the uncertainties in the model by frequently constraining the models solution with available observations (MalanotteRizzoli et al. 1989; Ghil 1989; Hoteit et al. 2012). When formulated as a Bayesian filtering problem, it can further provide estimates of the uncertainty on the final solution (Chassignet et al. 2018).

An efficient storm surge forecasting system should be able to assimilate the available data in real time to provide timely and reliable forecasts. The Kalman filter (KF), originally proposed by Kalman (1960) for linear state-space systems, is a popular Bayesian filtering approach for data assimilation. The implementation of the KF for data assimilation into large-scale storm surge models is, however, not possible because of their nonlinear nature and the prohibitive computational cost related to their large dimension. The ensemble Kalman filter (EnKF) was then introduced as a Monte Carlo-based implementation of the KF to tackle these challenges. The EnKF makes use of a set of realizations of model states, called ensemble, to estimate the first two moments, mean and covariance, of the KF in two steps (Evensen 2003): a forecast step that integrates the ensemble members forward with the model, and an analysis step to update the members with the incoming observations. The filter estimate and its error covariance are then taken as the sample mean and covariance of the ensemble. The (original) EnKF stochastically perturbs the observations before assimilation so that its analysis covariance asymptotically matches that of the KF (Burgers et al. 1998). This may, however, introduce sampling errors when the filter is implemented with small ensembles (smaller than the rank of the observational error), which generally leads to an underestimation of the analysis uncertainties (Hoteit et al. 2015). Various deterministic EnKFs were then proposed to avoid perturbing the observations, basically applying a KF analysis step to update the ensemble mean and a square root form of its sample error covariance (Tippett et al. 2003). These include the ensemble transform Kalman filter (ETKF) (Bishop et al. 2001), the ensemble adjustment Kalman filter (EAKF) (Anderson 2001), and the singular evolutive interpolated Kalman (SEIK) filters (Pham et al. 1998; Hoteit et al. 2002). Butler et al. (2012) and Altaf et al. (2013) demonstrated the relevance of SEIK for enhancing the short-range forecasting capabilities of the Advanced Circulation model (ADCIRC). Altaf et al. (2014) later compared the performances of the stochastic EnKF with several deterministic EnKFs and suggested that the latter can provide comparable performances with enough tuning, better than those of the stochastic EnKF, especially when implemented with small ensembles.

The representativeness of the forecast error covariance is crucial in a $\mathrm{KF}$ as this characterizes the structure of the analysis increment, and thus the efficiency of the update step (Lorenc 2003). The restricted ensemble sizes in realistic large-scale applications may, however, severely affect the accuracy of the EnKFs covariances, and therefore their solutions. Poorly specifying the model errors, which is common, would also result in underestimated filter covariances. Several approaches were introduced to enhance the representativeness of the ensemble forecast statistics, among which we cite; inflation (Anderson 2001), localization (Houtekamer and Mitchell 1998), hybrid covariance formulation (Hamill and Snyder 2000), robust filtering (Luo and Hoteit 2011), and the adaptive EnKF (Song et al. 2010). In particular, the hybrid EnKF estimates the background error covariance as a linear combination of a flow-dependent covariance estimated from the EnKF ensemble, and a time invariant (static) covariance typically used in an 
optimal interpolation (OI) or a three-dimensional variational data assimilation (3DVAR) system (Wang et al. 2009). The motivation behind this is to mitigate for the EnKF background limitations by complementing the ensemble-based error covariance with a preselected static covariance (Song et al. 2010).

Ensemble Kalman filtering with one-step-ahead (OSA) smoothing is another approach to enhance the performances of the EnKFs by constraining the sampling of the forecast ensemble with the future observation (AitEl-Fquih et al. 2016; Raboudi et al. 2018). In a linear Gaussian system, the analysis of an OSA-smoothed KF is identical to that of the KF (Desbouvries et al. 2011). In an ensemble framework, it was shown that the additional smoothing step enhances the filter solution, particularly in the cases of small ensembles, low spatial and temporal data coverage, and large model and observations errors (Raboudi et al. 2018). The smoothing step indeed enhances the representativeness of the forecast ensemble, which enables better exploitation of the observation. This is very similar to the Kalnay and Yang (2010) "running in place" algorithm (RIP), which was introduced to improve the EnKF behavior during periods of strong nonlinearities. This is expected to be particularly beneficial in the context of storm surge data assimilation, where EnKFs generally suffer during the strongly nonlinear surge periods (Altaf et al. 2013, 2014). However, the OSA smoothing scheme requires integrating the forecast ensemble twice, and thus doubles the overall computational cost. Since timely forecasts are a must in operational storm surge applications, we combine in this study the OSA smoothing scheme with the hybrid formulation to allow for an efficient implementation of the filter with small ensembles. We test the proposed framework with the ETKF to enhance the short-range storm surge forecasting capabilities of the ADCIRC model, using two storm surge test cases (Berg 2009; Torres et al. 2017).

The remainder of this paper is organized as follows. Section 2 provides an overview of the storm surge prediction model, ADCIRC, and section 3 provides an overview of the filtering schemes. Section 4 presents the experimental design, and analyzes and discusses the performances of the filters for forecasting the storm surge associated with Hurricane Ike. The conclusions are finally offered in section 5 .

\section{ADCIRC model}

Following the devastating 2005 hurricane season, a multi-institutional research team has been assigned the mission of developing and applying a state-of-the-art storm surge forecasting model (Luettich and Westerink 2004), for the purpose of simulating hydrodynamic circulations along shelves and coasts, and within estuaries. The ADCIRC model was then developed based on the shallow-water equations (SWEs). These are derived from the incompressible Navier-Stokes equations under the assumption of hydrostatic pressure and involve coupled generalized wave continuity equation and momentum equations (Lynch and Gray 1979). ADCIRC solves forms of the SWEs for water levels and vertically integrated momentum equations for water currents (Dawson et al. 2006; Luettich and Westerink 2004). It applies the continuous-Galerkin finite-element method with linear triangular elements to discretize and solve the SWEs on unstructured meshes, thereby allowing localized refinement in regions where the solution gradients are largest, and finite difference schemes in time. ADCIRC is further parallelized for distributed memory and multicore computers, which enables remarkable scalability on these platforms (Tanaka et al. 2011). In the particular case of storm surge applications, structural features such as levees, raised roads, and railways have to be included in the model configuration, and the model is primarily forced by tides, winds, and wind waves.

ADCIRC has an extensive and successful history of storm surge prediction applications in coastal waters and marginal seas (Butler et al. 2012), particularly in the Gulf of Mexico (Dietrich et al. 2011b). Data from previous storms dating from 1965 to 2008, including Hurricanes Betsy (1965), Katrina (2005), and Ike (2008) (Hope et al. 2013; Dietrich et al. 2011a; Kennedy et al. 2011) have been extensively used to validate ADCIRC. These data campaigns along with the fundamental knowledge gained from the hindcasting studies were very beneficial for the development of the real-time forecasting system, the ADCIRC Surge Guidance System (ASGS) (Luettich and Westerink 2007; Fleming et al. 2008). Interested readers are referred to Luettich and Westerink (2007) for full details about ADCIRC.

\section{Hybrid ETKF with one-step-ahead smoothing}

Consider the discrete-time dynamical system:

$$
\left\{\begin{array}{l}
\mathbf{x}_{n}=\mathscr{C l}_{n-1}\left(\mathbf{x}_{n-1}\right)+\eta_{n-1}, \\
\mathbf{y}_{n}=\mathbf{H}_{n} \mathbf{x}_{n}+\varepsilon_{n}
\end{array}\right.
$$

where $\mathbf{x}_{n} \in \mathbb{R}^{N_{x}}$ is the $N_{x}$ dimensional system state at time instant $n$ and $\mathbf{y}_{n} \in \mathbb{R}^{N_{y}}$ is the $N_{y}$ dimensional corresponding observation. The processes $\eta=\left\{\eta_{n}\right\}_{n \in \mathbb{N}}$ and $\varepsilon=\left\{\varepsilon_{n}\right\}_{n \in \mathbb{N}}$ represent the model and the observation noise, respectively, assumed to be independent in time, jointly independent, and Gaussian with zero means and covariances, $\mathbf{Q}_{n}$ and $\mathbf{R}_{n}$, respectively. The nonlinear 


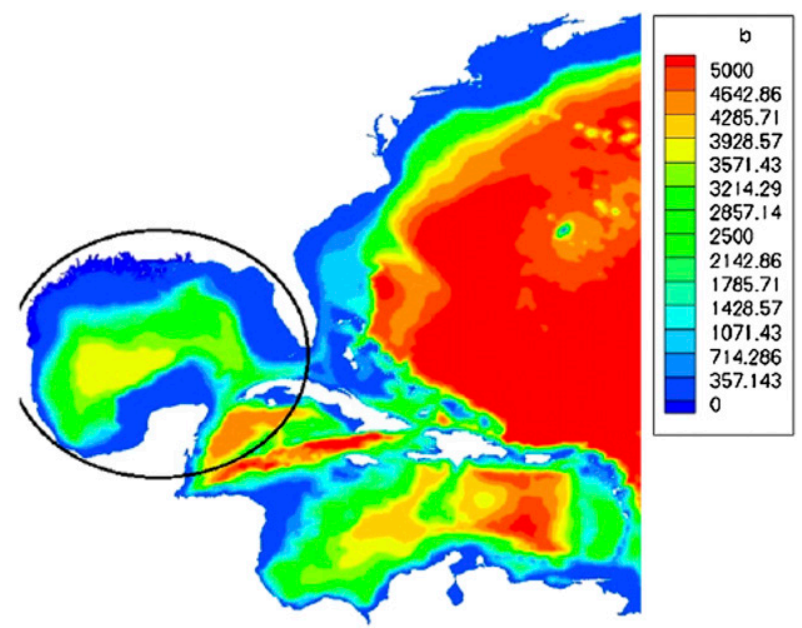

FIG. 1. Western North Atlantic domain and bathymetry (m). The Gulf of Mexico is circled in black (from Altaf et al. 2014).

dynamical model $\mathscr{A b}_{n-1}$ integrates the system state from time instant $n-1$ to $n$. $\mathbf{H}_{n}$ is the observation operator that projects $\mathbf{x}_{n}$ from the state space onto the observation space, which is linear in our setting [the case of nonlinear $\mathbf{H}_{n}$ could be treated as usually done in EnKFs; see, e.g., Liu et al. (2016)]. Here, we focus on the filtering problem, which consists of estimating the state, $\mathbf{x}_{n}$, at any time instant $n$, given all the observations up to time $n, \mathbf{y}_{0}, \mathbf{y}_{1}, \ldots, \mathbf{y}_{n}$. The posterior mean (PM), which minimizes the mean-squared error (MSE), is the standard solution for this problem (Ait-El-Fquih and Hoteit 2016).

For linear Gaussian systems, the KF provides a recursive computation of the PM. In the more general case of nonlinear $\mathscr{L}_{n-1}$, suboptimal EnKFs were proposed as Monte Carlo approximations of the KF that are particularly suitable for large dimensional applications.

\section{a. ETKF}

Let $\left\{\mathbf{x}_{n}^{f, i}\right\}_{i=1}^{N_{e}}$ denote an ensemble of $N_{e}$ forecast members at time instant $n$, and $\mathbf{P}_{\mathbf{x}_{n}^{f}}$ its sample covariance matrix. Let also $\mathbf{S}_{\mathbf{x}_{n}^{f}}$ be the forecast ensemble perturbation matrix with the $i$ th column defined as $\left(1 / \sqrt{N_{e}-1}\right)\left(\mathbf{x}_{n}^{f, i}-\overline{\mathbf{x}}_{n}^{f}\right)$, where $\overline{\mathbf{x}}_{n}^{f}$ is the ensemble mean [i.e., $\mathbf{P}_{\mathbf{x}_{n}^{f}}=\mathbf{S}_{\mathbf{x}_{n}^{f}}\left(\mathbf{S}_{\mathbf{x}_{n}^{f}}\right)^{\mathrm{T}}$ ]. Similar notations will be adopted hereafter for the smoothing, $\mathbf{x}_{n}^{s, i}$, and analysis, $\mathbf{x}_{n}^{a, i}$, ensembles. The observation $\mathbf{y}_{n}$ is used to update $\overline{\mathbf{x}}_{n}^{f}$ to obtain the analysis state $\overline{\mathbf{x}}_{n}^{a}$ as

$$
\begin{aligned}
\overline{\mathbf{x}}_{n}^{a}= & \overline{\mathbf{x}}_{n}^{f}+\mathbf{P}_{\mathbf{x}_{n}^{f}} \mathbf{H}_{n}^{\mathrm{T}}\left(\mathbf{H}_{n} \mathbf{P}_{\mathbf{x}_{n}^{f}} \mathbf{H}_{n}^{\mathrm{T}}+\mathbf{R}_{n}\right)^{-1}\left(\mathbf{y}_{n}-\mathbf{H}_{n} \overline{\mathbf{x}}_{n}^{f}\right) \\
= & \overline{\mathbf{x}}_{n}^{f}+\mathbf{S}_{\mathbf{x}_{n}^{f}}\left(\mathbf{H}_{n} \mathbf{S}_{\mathbf{x}_{n}^{f}}\right)^{\mathrm{T}}\left[\left(\mathbf{H}_{n} \mathbf{S}_{\mathbf{x}_{n}^{f}}\right)\left(\mathbf{H}_{n} \mathbf{S}_{\mathbf{x}_{n}^{f}}\right)^{\mathrm{T}}+\mathbf{R}_{n}\right]^{-1} \\
& \times\left(\mathbf{y}_{n}-\mathbf{H}_{n} \overline{\mathbf{x}}_{n}^{f}\right) .
\end{aligned}
$$

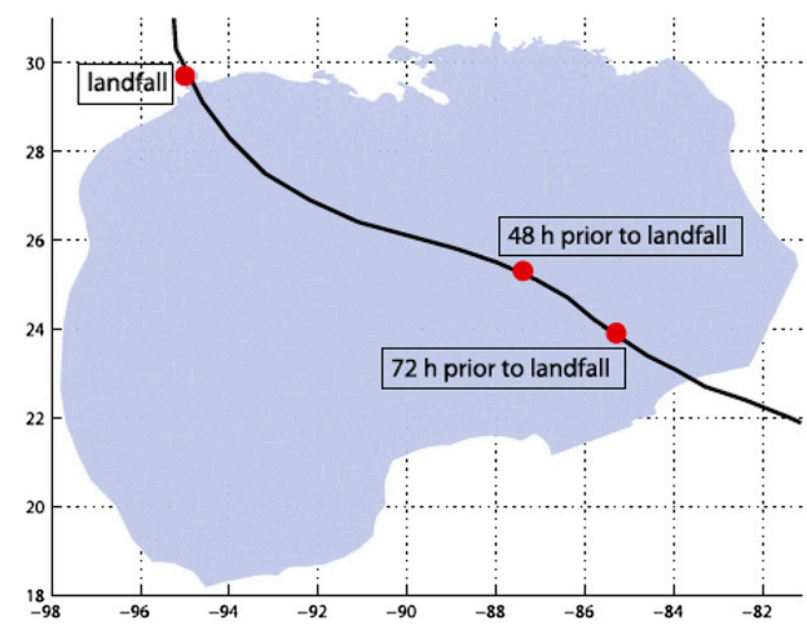

FIG. 2. Track of Hurricane Ike through the Gulf of Mexico. The circles with annotations are the locations of landfall at 0710 UTC13 Sep 2008 and the locations of the hurricane approximately 48 and $72 \mathrm{~h}$ before landfall. The $x$ axis and $y$ axis represent the longitude and the latitude in degrees, respectively.

The analysis error covariance matrix can be then expressed as $\mathbf{P}_{\mathbf{x}_{n}^{a}}=\mathbf{S}_{\mathbf{x}_{n}^{f}} \Phi_{n}\left(\mathbf{S}_{\mathbf{x}_{n}}\right)^{\mathrm{T}}$, where $\Phi_{n}=\left(\mathbf{I}+\mathbf{S}_{\mathbf{y}_{n}^{\prime}}^{\mathrm{T}} \mathbf{R}_{n} \mathbf{S}_{\mathbf{y}_{n}^{f}}\right)^{-1}, \mathbf{S}_{\mathbf{y}_{n}}=$ $\mathbf{H}_{n} \mathbf{S}_{\mathbf{x}_{n}^{f}}$, and $\mathbf{I}$ is the identity matrix. The ETKF updates the forecast error covariance matrix through forecast perturbation matrix. This is performed by postmultiplying $\mathbf{S}_{\mathbf{x}_{n}^{f}}$ by a transformation matrix $\mathbf{T}_{n}^{f}$ to obtain the analysis perturbations $\mathbf{S}_{\mathbf{x}_{n}^{a}}$ as

$$
\mathbf{S}_{\mathbf{x}_{n}^{a}}=\mathbf{S}_{\mathbf{x}_{n}^{f}} \mathbf{T}_{n}^{f} \mathbf{C}_{n}
$$

The matrix $\mathbf{T}_{n}^{f}$ is a square root of $\Phi_{n}$, computed via a singular value decomposition (SVD) of $\mathbf{S}_{\mathbf{y}_{n}^{\mathrm{T}}} \mathbf{R}_{n} \mathbf{S}_{\mathbf{y}_{n}^{f}}$ (Bishop et al. 2001; Wang et al. 2004):

$$
\mathbf{S}_{\mathbf{y}_{n}^{f}}^{\mathrm{T}} \mathbf{R}_{n} \mathbf{S}_{\mathbf{y}_{n}^{f}}=\mathbf{U} \boldsymbol{\wedge} \mathbf{U}^{\mathrm{T}},
$$

where $\mathbf{U}$ is a unitary matrix whose diagonal coefficients are the left singular vectors, and $\boldsymbol{\Lambda}$ is a diagonal matrix whose entries are the singular values. A square root of $\Phi_{n}$ is then obtained as $\mathbf{T}_{n}=\mathbf{U}(\mathbf{I}+\boldsymbol{\Lambda})^{-1 / 2} \cdot \mathbf{C}_{n}$ in (4) is a centering matrix satisfying $\mathbf{C}_{n} \mathbf{C}_{n}^{\mathrm{T}}=\mathbf{I}$ and $\mathbf{C}_{n} \mathbf{1}_{N_{e}}^{\mathrm{T}}=0$, where $\mathbf{1}_{N_{e}}$ is the $N_{e}$-dimensional ones vector (Wang et al. 2004). Several possible centering matrices have been suggested (see, e.g., Pham 2001; Bishop et al. 2001).

The analysis ensemble members are finally generated as

$$
\mathbf{x}_{n}^{a, i}=\overline{\mathbf{x}}_{n}^{a}+\sqrt{N_{e}-1}\left(\mathbf{S}_{\mathbf{x}_{n}^{a}}\right)_{i},
$$

where $\left(\mathbf{S}_{\mathbf{x}_{n}^{a}}\right)_{i}$ denotes the $i$ th column of $\mathbf{S}_{\mathbf{x}_{n}^{a}}$. 


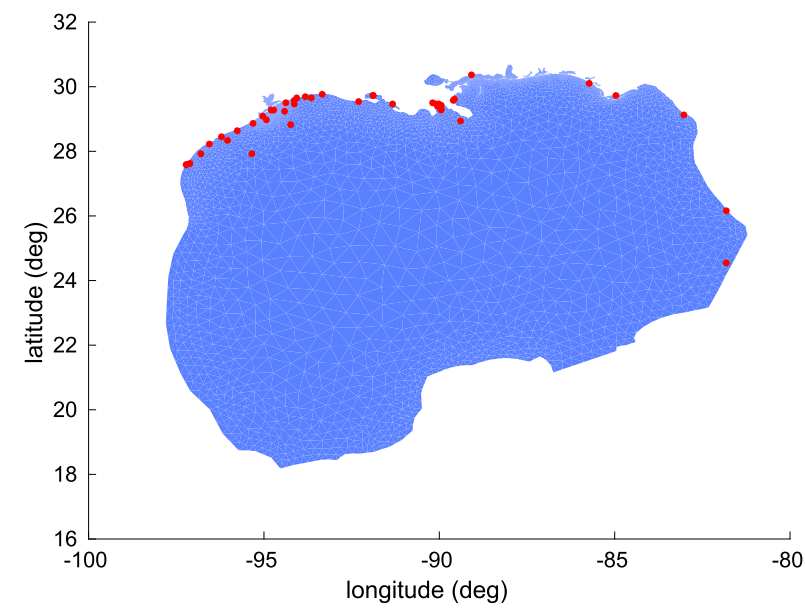

FIG. 3. The 43 observation stations used for Hurricane Ike simulations.

\section{b. ETKF-OSA algorithm}

Ensemble filtering with OSA smoothing applies a two-stage update step based on the same observation, for more efficient exploitation of the observations. Raboudi et al. (2018) proposed a deterministic version of the stochastic EnKF with OSA smoothing, based on the SEIK equations (SEIK-OSA). Here, we present a similar ETKF-OSA algorithm based on the ETKF.

\section{1) SMOOTHING STEP}

Starting from a forecast ensemble $\left\{\mathbf{x}_{n}^{f, i}\right\}_{i=1}^{N_{e}}$, ETKFOSA first computes the smoothed state estimate from the previous analysis as (Desbouvries et al. 2011):

$\overline{\mathbf{x}}_{n-1}^{s}=\overline{\mathbf{x}}_{n-1}^{a}+\mathbf{P}_{\mathbf{x}_{n-1}^{a}, \mathbf{y}_{n}^{f}}\left(\mathbf{H}_{n} \mathbf{P}_{\mathbf{x}_{n}^{f}} \mathbf{H}_{n}^{\mathrm{T}}+\mathbf{R}_{n}\right)^{-1}\left(\mathbf{y}_{n}-\mathbf{H}_{n} \overline{\mathbf{x}}_{n}^{f}\right)$,

where the cross-covariance term, $\mathbf{P}_{\mathbf{x}_{n-1}^{a}, \mathbf{y}_{n}^{f}}$, is evaluated from the ensemble members as

$$
\mathbf{P}_{\mathbf{x}_{n-1}^{a}, \mathbf{y}_{n}^{f}}=\mathbf{S}_{\mathbf{x}_{n-1}^{a}}\left(\mathbf{S}_{\mathbf{y}_{n}^{f}}\right)^{\mathrm{T}}
$$

The smoothed covariance can, in turn, be decomposed as $\mathbf{P}_{\mathbf{x}_{n-1}^{s}}=\mathbf{S}_{\mathbf{x}_{n-1}^{a}} \Phi_{n}\left(\mathbf{S}_{\mathbf{x}_{n-1}^{a}}\right)^{\mathrm{T}}$ and the smoothed members are finally obtained as in (6), using

$$
\mathbf{x}_{n-1}^{s, i}=\overline{\mathbf{x}}_{n-1}^{s}+\sqrt{N_{e}-1}\left(\mathbf{S}_{\mathbf{x}_{n-1}^{s}}\right),
$$

where $\left(\mathbf{S}_{\mathbf{x}_{n-1}^{s}}\right)_{i}$ denotes the $i$ th column of $\mathbf{S}_{\mathbf{x}_{n-1}^{s}}=$ $\mathbf{S}_{\mathbf{x}_{n-1}^{a}} \mathbf{T}_{n}^{f} \mathbf{C}_{n}$.

\section{2) ANALYSIS STEP}

The resulting smoothed ensemble is, again, integrated forward in time with the dynamical model to compute a

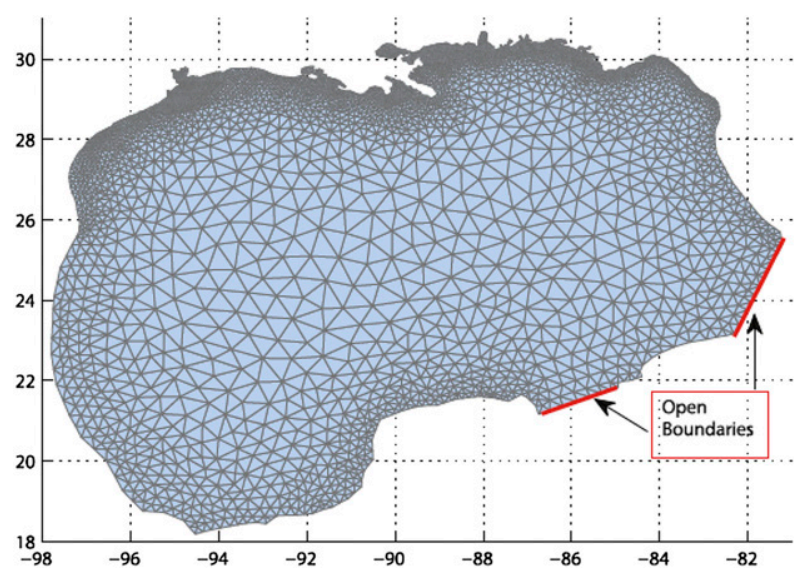

FIG. 4. Discretization of the Gulf of Mexico domain containing 8006 nodes and 14269 elements. Open boundaries are denoted by bold boundary lines. All other boundaries are land. The open boundaries are forced by the five tidal constituents: K1, O1, P1, M2, and S2. The land boundaries are reflective (from Altaf et al. 2014). The $x$ axis and $y$ axis represent the longitude and the latitude in degrees, respectively.

pseudo-forecast ensemble $\left\{\tilde{\mathbf{x}}_{n}^{f, i}\right\}_{i=1}^{N_{e}}$, which is then updated based on the observation $\mathbf{y}_{n}$ to compute the analysis as

$$
\overline{\mathbf{x}}_{n}^{a}=\overline{\tilde{\mathbf{x}}}_{n}^{f}+\mathbf{P}_{\tilde{\mathbf{x}}_{n}} \mathbf{H}_{n}^{\mathrm{T}}\left(\mathbf{H}_{n} \mathbf{P}_{\tilde{\mathbf{x}}_{n}^{f}} \mathbf{H}_{n}^{\mathrm{T}}+\mathbf{R}_{n}\right)^{-1}\left(\mathbf{y}_{n}-\mathbf{H}_{n} \overline{\tilde{\mathbf{x}}}_{n}^{f}\right) .
$$

The resampling of the analysis members of ETKF-OSA can be obtained following a similar approach as the analysis step of ETKF to get the analysis perturbations matrix $\mathbf{S}_{\mathbf{x}_{n}^{a}}$, with the only change of replacing the forecast members by the pseudo-forecast ones. The analysis ensemble is then sampled as

$$
\mathbf{x}_{n}^{a, i}=\overline{\mathbf{x}}_{n}^{a}+\sqrt{N_{e}-1}\left(\mathbf{S}_{\mathbf{x}_{n}^{a}}\right),
$$

where $\left(\mathbf{S}_{\mathbf{x}_{n}^{a}}\right)_{i}$ denotes the $i$ th column of $\mathbf{S}_{\mathbf{x}_{n}^{a}}$.

ETKF-OSA therefore involves two forecast steps with the dynamical model, the first to compute the forecast members and the second to compute the pseudo-forecast members, as well as two update steps; the first smooths the previous analysis mean and the corresponding error covariance matrix using the "future" observation, while the second updates, using the same observation, the pseudo-forecast estimate and its error covariance. This means that an EnKF with OSA smoothing is roughly twice more expensive than a standard ETKF. The hybrid formulation is thus used to allow for efficient implementation of the filter with small ensembles, as a straightforward way to reduce its computational cost.

\section{c. Hybrid formulation}

The hybrid ETKF $\left(\mathrm{ETKF}_{\mathrm{Hyb}}\right)$ forecast covariance is expressed as a linear combination of a flow-dependent 
TABLE 1. Outline of the differences between hindcast (truth) simulation to generate data, and data assimilation forecasting experiments.

\begin{tabular}{lll}
\hline \hline & \multicolumn{1}{c}{ Truth } & Data assimilation \\
\hline Domain & $\begin{array}{c}\text { Western North } \\
\text { Atlantic }\end{array}$ & Gulf of Mexico \\
Average mesh element size & $\begin{array}{c}1.34 \mathrm{~km}^{2} \\
\text { Time step }\end{array}$ & $\begin{array}{l}1 \mathrm{~s} \\
\mathrm{~km}^{2}\end{array}$ \\
Wind field & OWI & $10 \mathrm{~s}$ \\
Bottom friction formulation & Hybrid & Dynamic Holland \\
& & Chezy \\
\hline
\end{tabular}

ensemble covariance and a static background covariance:

$$
\mathbf{P}_{n}^{h}=(1-\alpha) \mathbf{P}_{\mathbf{x}_{n}^{f}}+\alpha \mathbf{B}^{x},
$$

where $0 \leq \alpha \leq 1, \mathbf{B}^{x}$ is a given static background covariance matrix, and $\mathbf{P}_{\mathbf{x}_{n}^{f}}$ is the flow dependent covariance estimated from an EnKF forecast ensemble. $\mathrm{ETKF}_{\mathrm{Hyb}}$ has the same algorithm as the ETKF, except for the use of $\mathbf{P}_{n}^{h}$ in the update step (2). In practice, the $N_{x} \times N_{x}$ matrix $\mathbf{P}_{n}^{h}$ is not explicitly computed, nor stored. Instead, the (cross)-covariance terms, $\mathbf{P}_{n}^{h} \mathbf{H}_{n}^{\mathrm{T}}$ and $\mathbf{H}_{n} \mathbf{P}_{n}^{h} \mathbf{H}_{n}^{\mathrm{T}}$, are computed based on a square root factorization, $\mathbf{S}_{B}$, of $\mathbf{B}^{x}$ (i.e., $\mathbf{B}^{x}=\mathbf{S}_{B} \mathbf{S}_{B}^{\mathrm{T}}$ ) as follows:

$$
\mathbf{P}_{n}^{h} \mathbf{H}_{n}^{\mathrm{T}}=(1-\alpha) \mathbf{S}_{\mathbf{x}_{n}^{f}}\left(\mathbf{H}_{n} \mathbf{S}_{\mathbf{x}_{n}^{f}}\right)^{\mathrm{T}}+\alpha \mathbf{S}_{B}\left(\mathbf{H}_{n} \mathbf{S}_{B}\right)^{\mathrm{T}},
$$

$\mathbf{H}_{n} \mathbf{P}_{n}^{h} \mathbf{H}_{n}^{\mathrm{T}}=(1-\alpha)\left(\mathbf{H}_{n} \mathbf{S}_{\mathbf{x}_{n}^{f}}\right)\left(\mathbf{H}_{n} \mathbf{S}_{\mathbf{x}_{n}^{f}}\right)^{\mathrm{T}}+\alpha\left(\mathbf{H}_{n} \mathbf{S}_{B}\right)\left(\mathbf{H}_{n} \mathbf{S}_{B}\right)^{\mathrm{T}}$.

$\mathbf{S}_{B}$ is often obtained from a large inventory of historical system states sampled by the dynamical model (Song et al. 2010; Tsiaras et al. 2017; Toye et al. 2017). The hybridized covariances are exclusively used to update the forecast ensemble mean and compute the hybrid analysis mean $\overline{\mathbf{x}}_{n}^{a, h}$. The resampling of the analysis members is implemented following the standard ETKF, based on $\overline{\mathbf{x}}_{n}^{a, h}$ and $\mathbf{S}_{\mathbf{x}_{n}^{f}}$ [i.e., similarly to (6) but after replacing $\overline{\mathbf{x}}_{n}^{a}$, by $\left.\overline{\mathbf{x}}_{n}^{a, h}\right]$.

The $\mathrm{ETKF}_{\text {hyb }}$-OSA algorithm is the hybrid version of ETKF-OSA, and thus involves two update steps, each based on a hybrid covariance formulation. It only differs from ETKF-OSA in the computation of the smoothed and analysis estimates.

\section{1) SMoothing STeP}

The smoothing step as presented in (7) of ETKF-OSA involves not only the covariance $\mathbf{P}_{\mathbf{x}_{n}^{f}}$, but also the cross covariance, $\mathbf{P}_{\mathbf{x}_{n-1}^{a}, \mathbf{y}_{n}^{f}}$, between the previous analysis perturbations and the current observational forecast

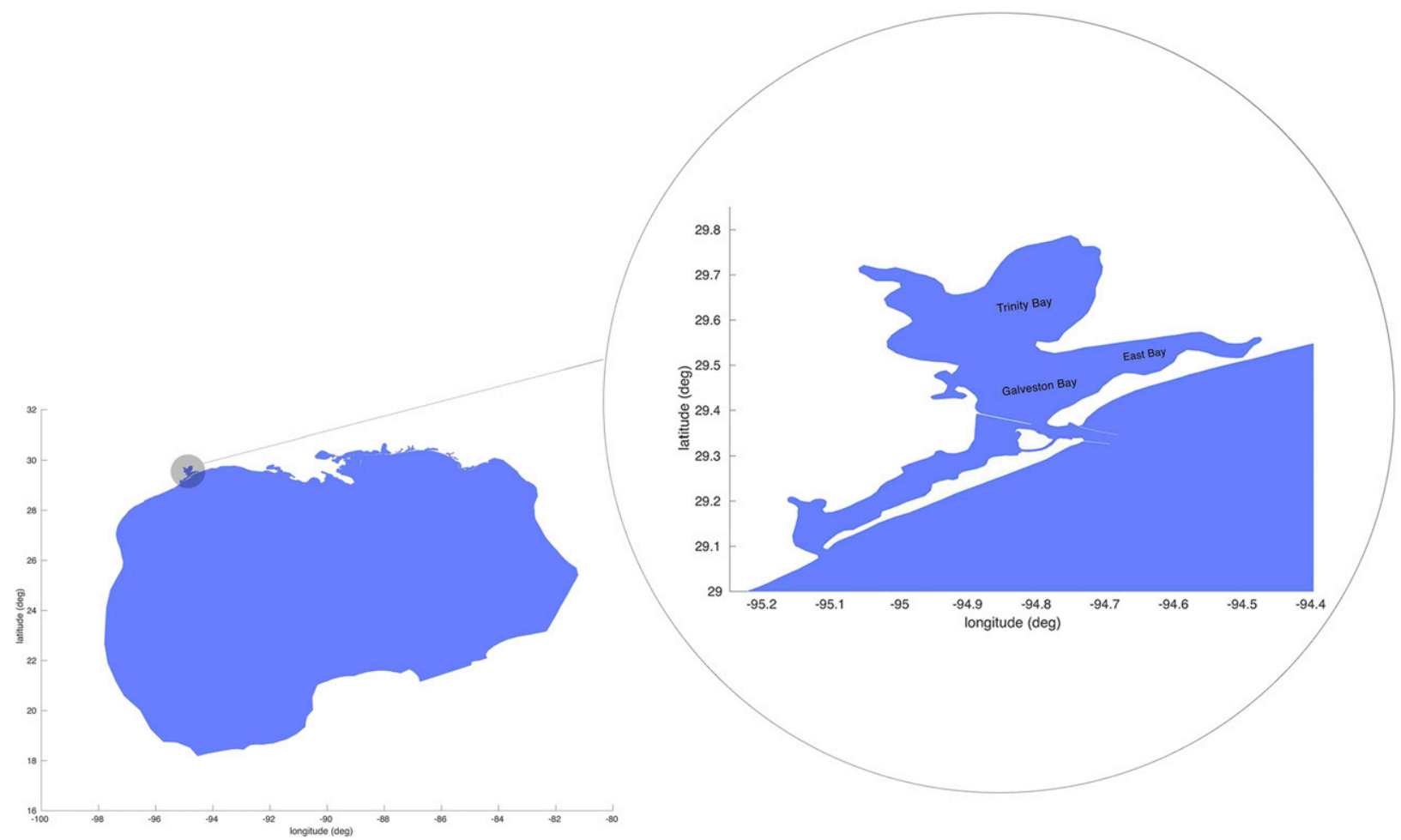

FIG. 5. Coastal regions which witnessed the largest surges $\left(29^{\circ}-29.8^{\circ} \mathrm{N}, 94.4^{\circ}-95.25^{\circ} \mathrm{W}\right)$. 

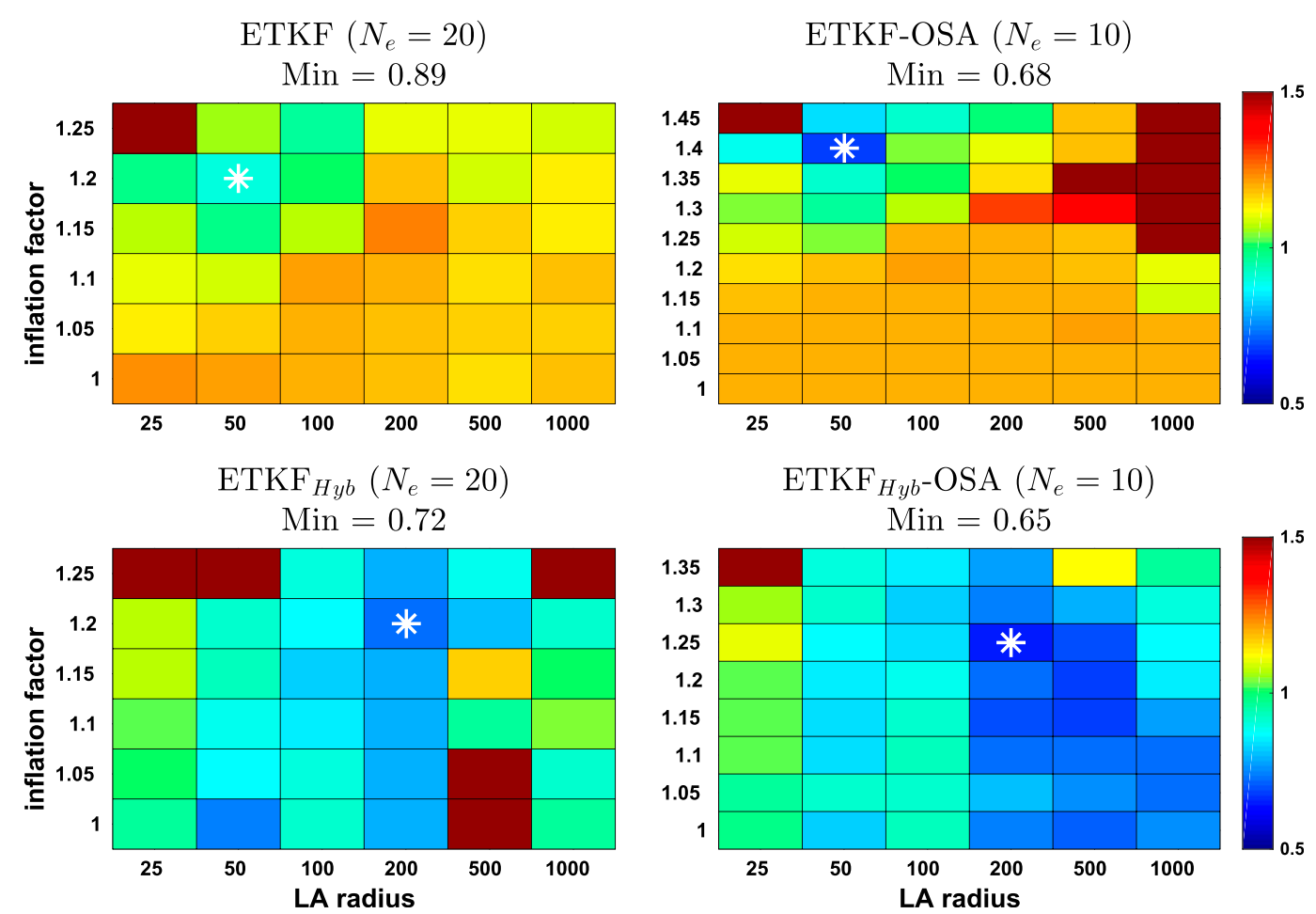

FIG. 6. Coastal-averaged RMSEs (m) of maximum water elevation forecasts for different LA radii and inflation factors using ETKF and $\mathrm{ETKF}_{\mathrm{Hyb}}$ with 20 members and ETKF-OSA and $\mathrm{ETKF}_{\mathrm{Hyb}^{-}} \mathrm{OSA}$ with 10 members. The asterisks indicate the minimum RMSE (given in the titles).

perturbations. Thus, to hybridize the smoothing Kalman gain, one needs to build, in addition to the aforementioned static covariance $\mathbf{B}^{x}$, another static cross covariance $\mathbf{B}_{\delta n}^{x y}$ between the state perturbations at a given time step and the observational forecast perturbations at the following time step. Practically, $\mathbf{B}_{\delta n}^{x y}$ can be computed using the same sequence of free-run model outputs used to compute $\mathbf{B}^{x}$, but considering the covariances between the states perturbations' and the "shifted in time" observational forecast perturbations. If $\mathbf{H}_{n}$ is constant in time (which is the case in our numerical experiments), these computations are done once, stored, and used during the whole simulation. One can then define a hybrid cross covariance as

$$
\mathbf{P}_{\mathbf{x}_{n-1}^{a}, \mathbf{y}_{n}^{f}}^{h}=\left(1-\alpha^{s}\right) \mathbf{P}_{\mathbf{x}_{n-1}^{a}, \mathbf{y}_{n}^{f}}+\alpha^{s} \mathbf{B}_{\delta n}^{x y},
$$

and a hybrid forecast error covariance, in a similar way as in the $\mathrm{ETKF}_{\mathrm{Hyb}}$ algorithm:

$$
\mathbf{P}_{n}^{h}=\left(1-\alpha^{f}\right) \mathbf{P}_{\mathbf{x}_{n}^{f}}+\alpha^{f} \mathbf{B}^{x}
$$

Substituting (16) and (15) in (7) leads to a smoothing mean of the following form:

$$
\overline{\mathbf{x}}_{n-1}^{s, h}=\overline{\mathbf{x}}_{n-1}^{a}+\mathbf{P}_{\mathbf{x}_{n-1}^{a}, \mathbf{y}_{n}^{f}}^{h}\left(\mathbf{H}_{n} \mathbf{P}_{n}^{h} \mathbf{H}_{n}^{\mathrm{T}}+\mathbf{R}_{n}\right)^{-1}\left(\mathbf{y}_{n}-\mathbf{H}_{n} \overline{\mathbf{x}}_{n}^{f}\right)
$$

where

$$
\begin{aligned}
& \mathbf{P}_{\mathbf{x}_{n-1}^{a}, \mathbf{y}_{n}^{f}}^{h}=\left(1-\alpha^{s}\right) \mathbf{S}_{\mathbf{x}_{n-1}^{a}}\left(\mathbf{H}_{n} \mathbf{S}_{\mathbf{x}_{n}^{f}}\right)^{\mathrm{T}}+\alpha^{s} \mathbf{B}_{\delta n}^{x y} \\
& \mathbf{H}_{n} \mathbf{P}_{n}^{h} \mathbf{H}_{n}^{\mathrm{T}}=\left(1-\alpha^{f}\right)\left(\mathbf{H}_{n} \mathbf{S}_{\mathbf{x}_{n}^{f}}\right)\left(\mathbf{H}_{n} \mathbf{S}_{\mathbf{x}_{n}^{f}}\right)^{\mathrm{T}}+\alpha^{f}\left(\mathbf{H}_{n} \mathbf{S}_{B}\right)\left(\mathbf{H}_{n} \mathbf{S}_{B}\right)^{\mathrm{T}}
\end{aligned}
$$

The smoothed ensemble is finally computed as in (9), but based on $\overline{\mathbf{x}}_{n-1}^{s, h}$ in (17).

\section{2) ANALysis STEP}

In the analysis step, the pseudo-forecast covariance is decomposed as in the forecast step:

$$
\tilde{\mathbf{P}}_{n}^{h}=(1-\tilde{\alpha}) \mathbf{P}_{\tilde{\mathbf{x}}_{n}^{f}}+\tilde{\alpha} \mathbf{B}^{x} .
$$

The analysis mean is consequently obtained as

$$
\overline{\mathbf{x}}_{n}^{a, h}=\overline{\tilde{\mathbf{x}}}_{n}^{f}+\tilde{\mathbf{P}}_{n}^{h} \mathbf{H}_{n}^{\mathrm{T}}\left(\mathbf{H}_{n} \tilde{\mathbf{P}}_{n}^{h} \mathbf{H}_{n}^{\mathrm{T}}+\mathbf{R}_{n}\right)^{-1}\left(\mathbf{y}_{n}-\mathbf{H}_{n} \overline{\tilde{\mathbf{x}}}_{n}^{f}\right),
$$

where $\tilde{\mathbf{P}}_{n}^{h} \mathbf{H}_{n}^{\mathrm{T}}$ and $\mathbf{H}_{n} \tilde{\mathbf{P}}_{n}^{h} \mathbf{H}_{n}^{\mathrm{T}}$ are given by (13) and (14) after replacing $\mathbf{P}_{n}^{h}$ by $\tilde{\mathbf{P}}_{n}^{h}$ and $\mathbf{S}_{\mathbf{x}_{n}^{f}}$ by $\mathbf{S}_{\tilde{\mathbf{x}}_{n}^{f}}$. The resampling of 

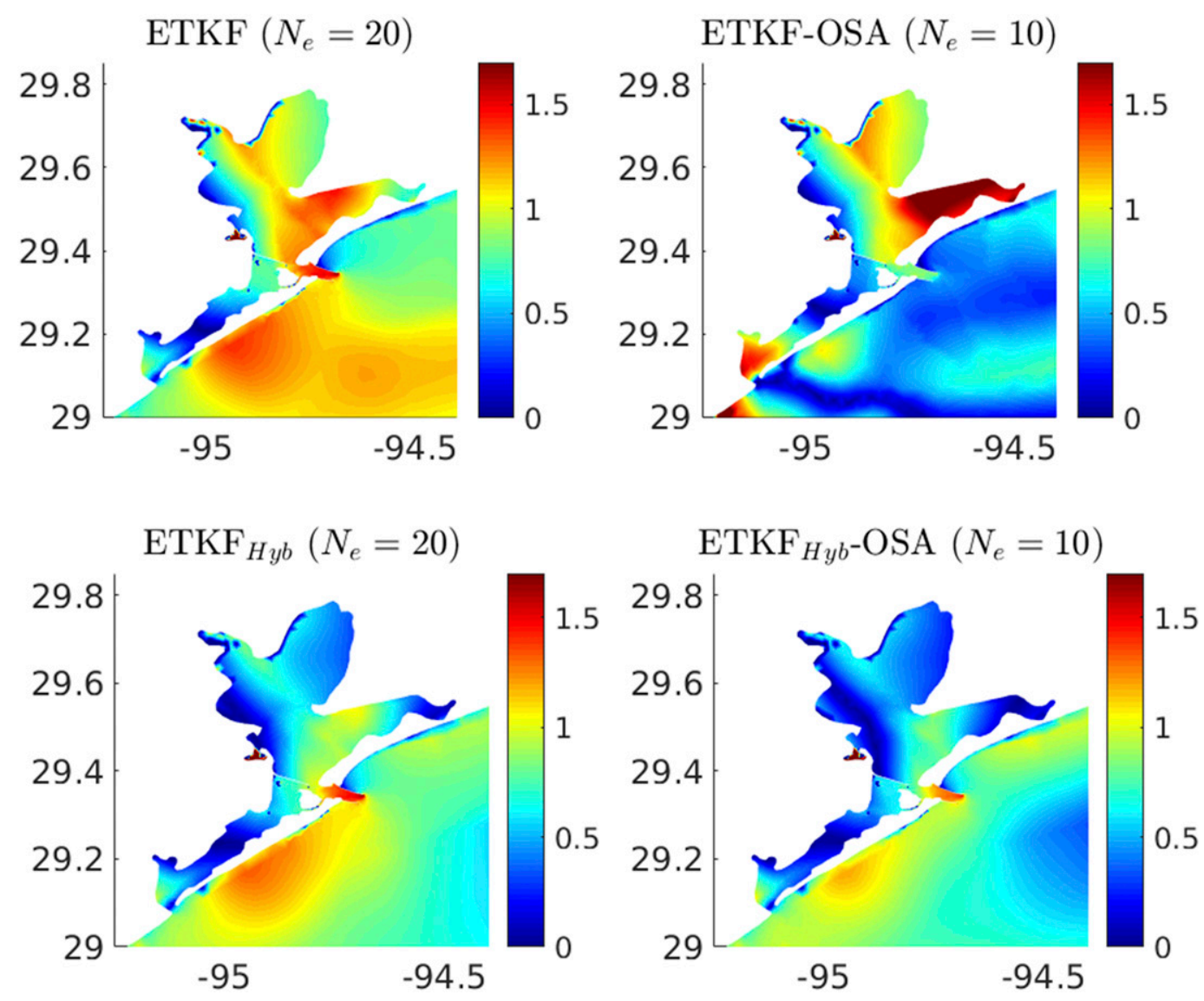

FIG. 7. Error $(\mathrm{m})$ between maximum free-surface elevation predictions and maximum true state. The results are presented for the best configurations (indicated by the asterisks in Fig. 6) of ETKF and ETKF $\mathrm{Hyb}_{\mathrm{b}}$ with 20 members and ETKF-OSA and ETKF $\mathrm{Hyb}_{\mathrm{b}} \mathrm{OSA}$ with 10 members.

the analysis ensemble is then performed as in (11), but using $\overline{\mathbf{x}}_{n}^{a, h}$ given by (21).

\section{Assimilation experiments and results}

Numerical experiments are conducted using the ADCIRC model configured for a storm surge forecasting problem over a domain including the Gulf of Mexico coastline (Fig. 1) and using the Hurricane Ike event as a test case (Berg 2009). We have also conducted another set of similar assimilation experiments based on what is known as Storm 36. This intense storm was generated as part of FEMA's latest comprehensive floodplain remapping study for the U.S. Gulf Coast, using the modified Joint Probability Method with an Optimal Sampling (JPM-OS) approach (Torres et al. 2017). Our assimilation results with Storm 36 were quite similar to those reported for Ike, and are thus not shown. In September 2008, Ike traveled through the Atlantic, Caribbean, and Gulf of Mexico to finally make landfall along the upper Texas coast at 0710 UTC 13 September
2008 (see Fig. 2). Upon making landfall, it was classified as a category 2 hurricane, after reaching a category 4 on 4 September 2008 (Berg 2009). Hurricane Ike was the third costliest hurricane ever to make landfall in the United States, being responsible for roughly 200 deaths (Berg 2009).

We follow Butler et al. (2012) and Altaf et al. (2014) and conduct twin experiments in the Gulf of Mexico considering two different configurations of the ADCIRC model. The first configuration, referred to as the reference run, is used to generate the reference states from which synthetic observations are extracted, while the second configuration is used as the forecast model in the data assimilation experiments.

\section{a. ADCIRC configurations}

To generate the reference states, ADCIRC was first configured on a domain covering the Gulf of Mexico and the western North Atlantic seaboard (see Fig. 1) with a high-resolution grid of 3322439 nodes corresponding to 6615381 elements and a time step of $1 \mathrm{~s}$. The model was 

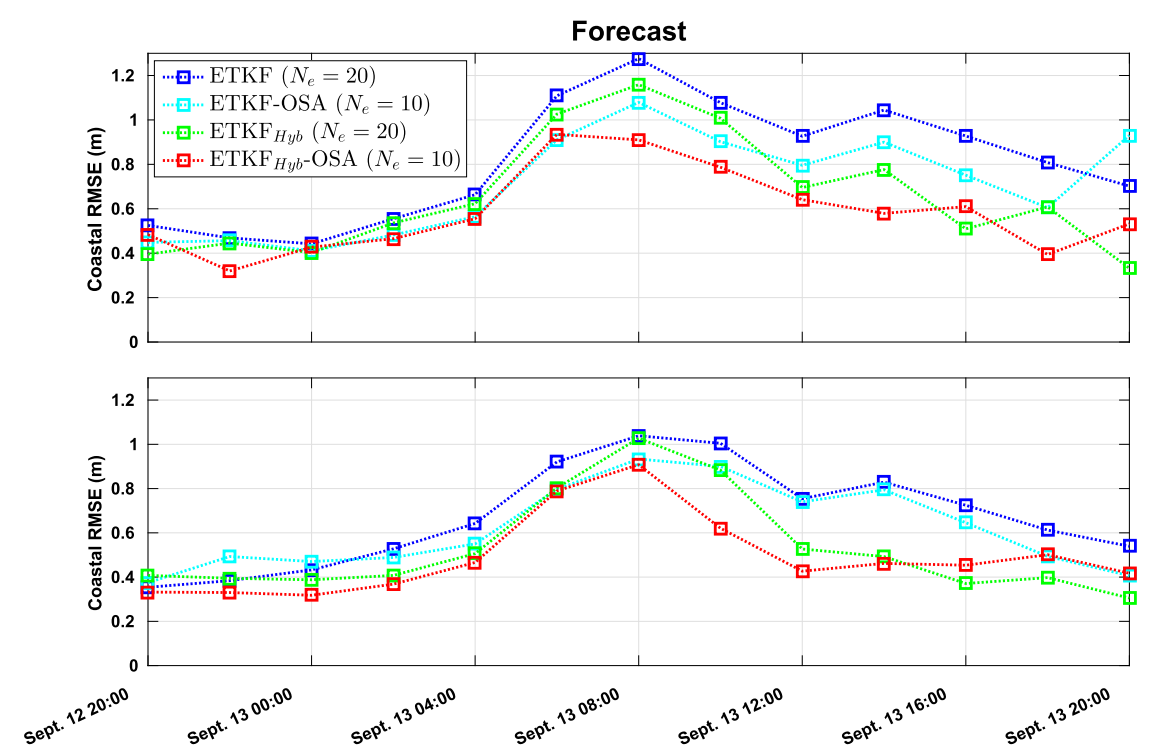

FIG. 8. Coastal-averaged RMSE of water elevations (in $\mathrm{m}$ ). The results are presented for the best configurations of the ETKF and ETKF $_{\mathrm{Hyb}}$ with 20 members and ETKF-OSA and ETKF $_{\mathrm{Hyb}}$-OSA with 10 members. The landfall took place at around 0700 UTC 13 Sep.

forced with high-fidelity wind fields that were generated from wind data collected during the actual hurricane and with data-assimilated atmospheric pressure fields provided by the Ocean Weather, Inc. (OWI). Assimilated observations of water levels were extracted and stored every $2 \mathrm{~h}$, according to 43 observation stations corresponding to actual measurement sites that were placed by several federal agencies, including the U.S. Geological Survey (USGS), NOAA, and some university research groups. The locations of the 43 stations are depicted in Fig. 3. The extracted data have been shown to match well the actual observations from these instruments for a couple of recent hurricanes (Butler et al. 2012). These stations are all located near the coasts where a coarse model generally tends to underestimate the recorded surge data (Altaf et al. 2014). The assimilation experiments would then assess the ability of this existing observation network to improve the short-range forecasting skill of a forecast model.

For the assimilation experiments, ADCIRC was configured on a coarser-resolution grid of 8006 nodes, corresponding to 14269 elements, covering only the Gulf of Mexico as shown in Fig. 4, and a model time step of $10 \mathrm{~s}$. The model is forced with coarse global wind fields generated by the dynamic Holland wind model using the best possible hurricane track data obtained from the National Oceanic and Atmospheric Administration (NOAA) archive (ftp://ftp.tpc.ncep.noaa.gov/atcf/archive/) (Holland 1980). This is quite different from the wind used to force the reference run in order to mimic a realistic storm surge exercise where the wind fields are not perfectly known. This would most likely be the case in an actual hurricane event as the best track wind data that would be available will certainly still be subject to uncertainties. Table 1 summarizes the main differences between ADCIRC configurations of the reference and assimilation runs.

\section{b. Assimilation experiments}

The data assimilation experiments follow Butler et al. (2012) and Altaf et al. (2014). After 1 day spinup starting at 0000 UTC 9 September 2008, observations extracted from the reference run according to the 43 observation stations network are assimilated every $2 \mathrm{~h}$ until at 0600 UTC 14 September 2008, 1 day after Hurricane Ike landfall, resulting in 51 assimilation steps. The observations noise are assumed independent with standard deviation $\sigma=0.03 m$ (i.e., $\mathbf{R}_{n}=\sigma^{2}$ I). The initial ensemble is generated using a second-order exact sampling based on an empirical orthogonal function (EOF) analysis (Hoteit et al. 2013). The ADCIRC model was first driven only by tidal forcing for 60 days, to eliminate all transient behavior, and the model state was saved every $5 \mathrm{~h}$ for a representative dataset. An EOF analysis was then applied on this dataset, based on which 9 EOF modes were retained to describe $90 \%$ of the total variance of the dataset. Hence, the initial ensemble is computed using a covariance from a physically representative space. A total of 10 ensemble members were then generated whose sample covariance matches the EOF-truncated covariance of the dataset. 


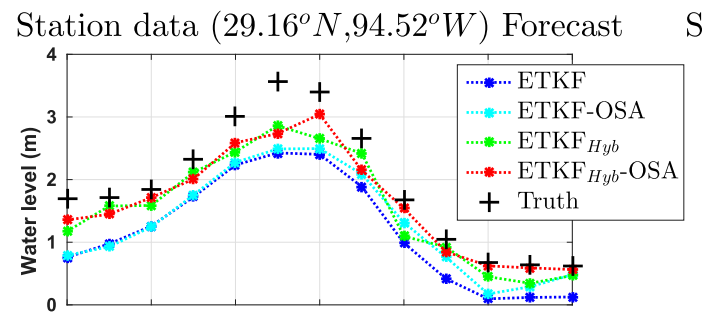

Station data $\left(29.16^{\circ} N, 94.52^{\circ} W\right)$ Analysis

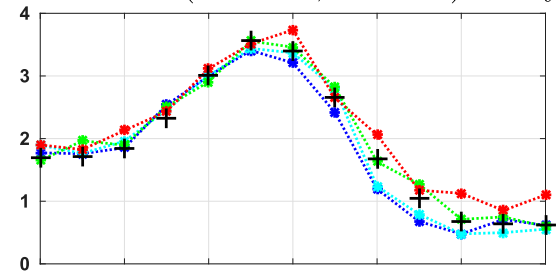

Station data $\left(29.28^{\circ} N, 94.9^{\circ} W\right)$ Forecast

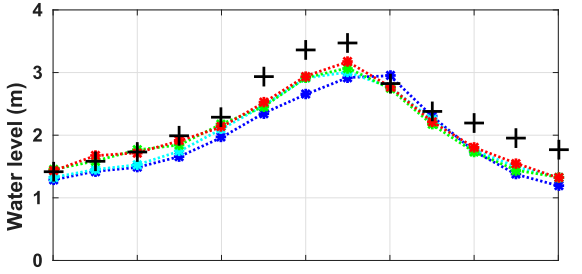

Station data $\left(29.28^{\circ} N, 94.9^{\circ} W\right)$ Analysis

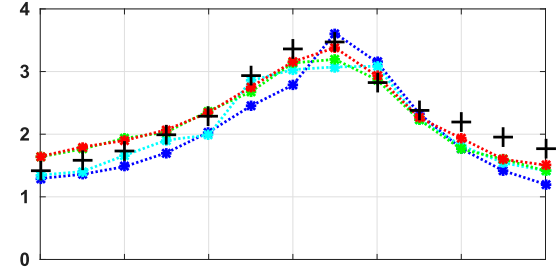

Station data $\left(29.66^{\circ} N, 94.81^{\circ} \mathrm{W}\right)$ Forecast

Station data $\left(29.66^{\circ} N, 94.81^{\circ} \mathrm{W}\right)$ Analysis
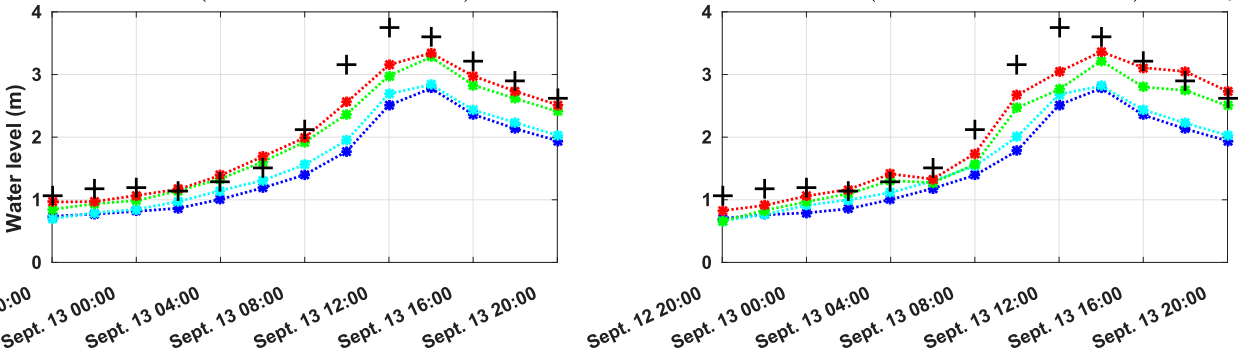

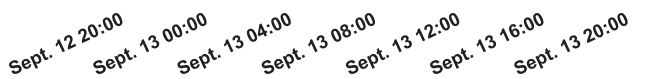

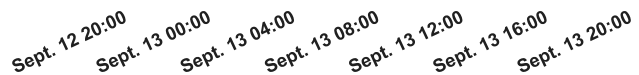

FIG. 9. Plots (in $\mathrm{m}$ ) of true states [(+) marks] vs filters' forecasts. Forecast and analysis results are presented for the best configurations of the ETKF and ETKF $_{\mathrm{Hyb}}$ with 20 members and ETKF-OSA and ETKF $\mathrm{Hyb}-\mathrm{OSA}$ with 10 members.

With the same (flow-dependent) ensemble size, the hybrid formulation only marginally increases the computational cost compared to a standard EnKF. The OSAsmoothing EnKFs are roughly twice more demanding than their standard counterparts, as they involve two forecast steps and two update steps. We therefore chose to implement and compare the OSA-smoothing ETKFs (ETKF-OSA and ETKF $_{\mathrm{Hyb}^{-}}$-OSA) with only half the ensemble size of their standard counterparts (ETKF and $\mathrm{ETKF}_{\mathrm{Hyb}}$ ) in all our assimilation experiments. Our goal is indeed not to compare the schemes using the same number of dynamic members, but rather to assess their performances under comparable computational costs. As expected, the hybrid schemes only marginally increase the computation costs compared to the nonhybrid schemes. Besides, the computing times of

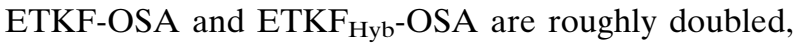
compared to ETKF and $\mathrm{ETKF}_{\mathrm{Hyb}}$, respectively, for the same ensemble size, but are comparable when the OSA smoothing schemes are implemented with half the number of dynamic ensemble members. Local analysis was implemented in all filters to update each grid point using only observations falling within a preset influence radius (Sakov and Bertino 2011; Houtekamer and Mitchell 1998). In our experiments, the localization support radii vary between 25 and $1000 \mathrm{~km}$. The filters were also equipped with the covariance inflation technique, which is commonly used to increase the spread of the forecast or analysis ensembles. Luo and Hoteit (2011) followed a robust filtering strategy and interpret it as an EnKF equipped with different ensemble inflation techniques. They argued that inflating the analysis ensemble enhances EnKFs performances compared to inflating the forecast ensemble, which was latter supported by Altaf et al. (2013) based on storm surge forecasting experiments using a SEIK filter with ADCIRC. Similar conclusions were also made by Wang and Bishop (2003). In the experiments presented in section $4 \mathrm{c}$, the analysis ensemble covariance was inflated by a factor $\lambda^{2}$. The filters were tested with different values of the inflation factor $\lambda$. The smoothed members of ETKF-OSA and ETKF $_{\mathrm{Hyb}}-\mathrm{OSA}$ were also inflated by a factor of 1.05 after several trial-and-error experiments. The weight of the stationary covariance in the hybrid covariance formulation is determined by trialand-error experiments based on which $\alpha$ (in $\mathrm{ETKF}_{\mathrm{Hyb}}$ ), 

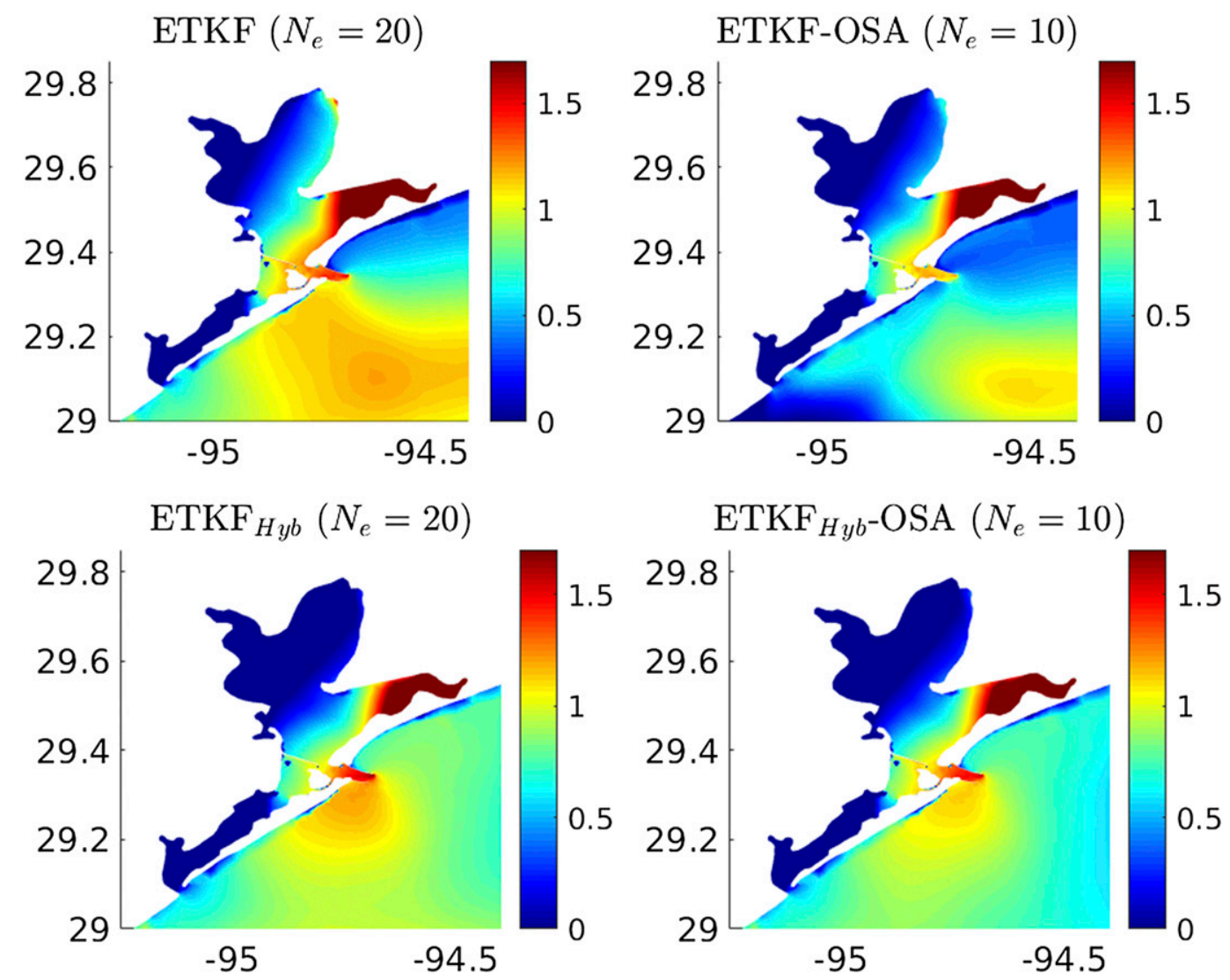

FIG. 10. Free surface elevation error (in m) at 0600 UTC 13 Sep 2008, one hour before the landfall from the truth. The results are presented for the best configurations of the ETKF and ETKF ${ }_{\mathrm{Hyb}}$ with 20 members and ETKF-OSA and $\mathrm{ETKF}_{\mathrm{Hyb}}$-OSA with 10 members.

$\alpha^{f}$ (in the first forecast of $\mathrm{ETKF}_{\mathrm{Hyb}}-\mathrm{OSA}$ ), and $\tilde{\alpha}$ (in the second forecast of $\mathrm{ETKF}_{\mathrm{Hyb}}$-OSA) were all set to 0.4. The results of these experiments suggested that the filters' behavior is not very sensitive to the choice of the weighting factors of the stationary covariance for values around 0.4 , while reducing their values to less than 0.1 resulted in hybrid schemes performances close to those of the standard filters. Assigning larger weights to the stationary covariance (above 0.6 ) also reduced the benefit of the hybrid scheme, which became less robust to the choice of the inflation factor and localization length scale. The other hybrid cross-covariance weight $\alpha^{s}$ in the smoothing step of $\mathrm{ETKF}_{\mathrm{Hyb}}$-OSA was also tuned by trial and error to 0.05 . Such a small value of $\alpha^{s}$ was required to scale the static cross covariance $\mathbf{B}_{\delta n}^{x y}$ to the flow-dependent cross covariance $\mathbf{P}_{\mathbf{x}_{n-1}^{a}, \mathbf{y}_{n}^{f}}$.

The most important objective of a storm surge forecasting system is to provide reliable predictions of the maximum surge along the coast (Butler et al. 2012; Altaf et al. 2013). We therefore evaluate the filters performances by their ability to predict the maximum storm surge based on the average root-mean-square error
(RMSE) of the maximum water elevation predictions over the duration of the storm and along the coastal regions of the largest surges $\left(29^{\circ}-29.8^{\circ} \mathrm{N}, 94.4^{\circ}-95.25^{\circ} \mathrm{W}\right)$ as indicated in Fig. 5. Predicting the storm surge at certain specific times, particularly in the few hours preceding the hurricane landfall, is also of interest and will also be assessed.

\section{c. Results}

Figure 6 plots the average RMSEs of the maximum water level forecasts for the Ike simulations as resulting from the four filters with an ensemble of 20 members for ETKF and ETKF $_{\mathrm{Hyb}}$ and 10 members for their OSAsmoothing counterparts, and different values of inflation factors and localization radii. Overall, both ETKF-OSA and $\mathrm{ETKF}_{\mathrm{Hyb}}$ improve the RMSE of maximum water elevation forecasts by almost $23 \%$ and $19 \%$ compared to the standard ETKF, respectively. The ETKF achieves its lowest RMSE (0.89) using an inflation factor of 1.2 and a localization radius of $50 \mathrm{~km}$. ETKF $\mathrm{Hyb}_{\mathrm{b}}$ reduces this error to 0.72 using an inflation factor of 1.2 and a localization radius of $200 \mathrm{~km}$, while with ETKF-OSA, 

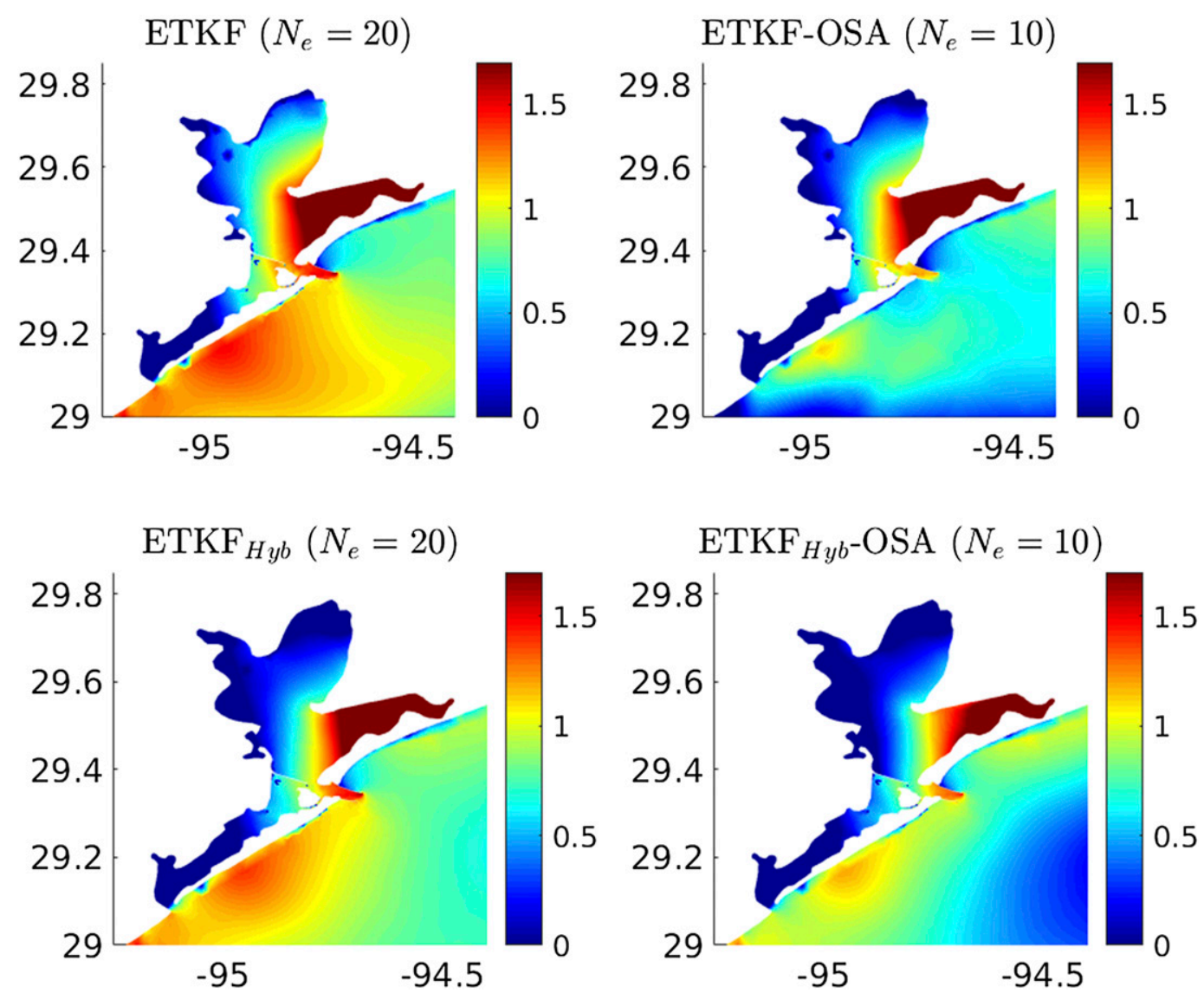

FIG. 11. Free surface elevation error (in m) at 0800 UTC 13 Sep 2008, one hour after the landfall from the truth. The results are presented for the best configurations of the ETKF and $\mathrm{ETKF}_{\mathrm{Hyb}}$ with 20 members and by ETKF-OSA and ETKF $\mathrm{Hyb}_{\mathrm{H}} \mathrm{OSA}$ with 10 members.

this error reduces to 0.68 using an inflation factor of 1.4 and a localization radius of $50 \mathrm{~km}$. ETKF $_{\mathrm{Hyb}} \mathrm{OSA}$ provides the lowest RMSE (0.65) using an inflation factor of 1.25 and a localization radius of $200 \mathrm{~km}$, which suggests a significant reduction of the RMSE by more than $26 \%$ compared to the ETKF. The hybrid ETKFs seem to require less localization to achieve their minimum RMSEs $\left(200 \mathrm{~km}\right.$ for both $\mathrm{ETKF}_{\mathrm{Hyb}}$ and $\mathrm{ETKF}_{\mathrm{Hyb}}-\mathrm{OSA}$ compared to $50 \mathrm{~km}$ for ETKF and ETKF-OSA), and are also more robust to the choice of localization radii. ETKF-OSA also requires larger inflation $(\lambda=1.4)$ compared to the standard ETKF $(\lambda=1.2)$. This is likely because the two update steps in the OSA-smoothing filter tend to further reduce the spread of the ensemble.

The averaged RMSE of maximum water level forecasts only provides a summary statistic of the estimation errors, but does not indicate the time or location where these errors actually occur. One should thus also be interested in analyzing the spatial distribution of the errors of maximum water level forecasts along the coastal area as well as the errors in water elevations in the few hours that precede the surge. We therefore present in Fig. 7 the spatial distribution of the errors for the maximum water elevations as resulting from the different filters using their best combinations of inflation and localization. $\mathrm{ETKF}_{\mathrm{Hyb}} \mathrm{OSA}$, and, to a lesser degree, ETKFOSA and $\mathrm{ETKF}_{\mathrm{Hyb}}$, perform significantly better than ETKF over most parts of the coastal domain. In particualr, ETKF-OSA produces significantly better estimates, except at few local areas (East Bay, Galveston Bay, and Trinity Bay as shown in Fig. 5). This might be related to the fact that ETKF and ETKF-OSA require stronger localization $(50 \mathrm{~km})$ than the hybrid schemes $(200 \mathrm{~km})$. With such a small localization radius, these areas are almost not affected by the update step. The hybrid schemes, however, enable larger localization radii, thereby broadening the influence of the available observations and extending the forecast update to those areas.

Figure 8 plots the forecast and analysis RMSEs for the water elevation evaluated over the landfall area as resulting from the different filters. We limit the analysis to 

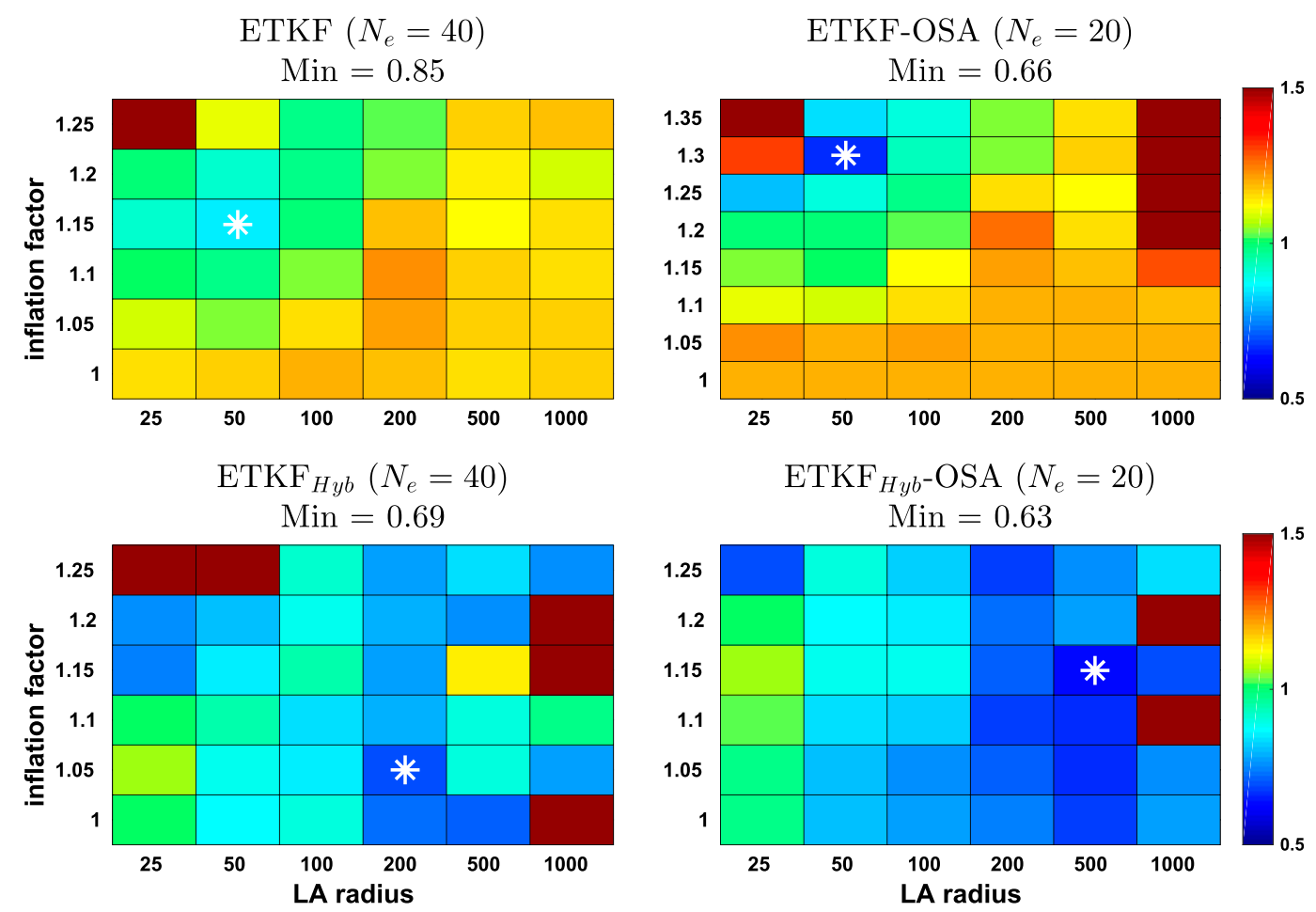

FIG. 12. As in Fig. 6, but using 40 members with ETKF and ETKF $_{\mathrm{Hyb}}$, and 20 members with ETKF-OSA and $\mathrm{ETKF}_{\mathrm{Hyb}}-\mathrm{OSA}$.

the time interval between 2000 UTC 12 September and 2000 UTC 13 September 2008 to focus on the landfall period. Overall, and compared to the ETKF, OSAsmoothing, and hybrid schemes tend to reduce the errors of water level forecasts after assimilation, mainly during the surge and in the few hours that precede the landfall. This is an important result as producing more accurate short-range forecasts of the water surge is our main objective. Before and during the passage of a hurricane, the modeled system exhibits a change of regime, which causes further uncertainties in the filtering process (Bennett 1992). During such periods of strong nonlinearities, the performances of KF-based schemes are known to degrade (Hoteit et al. 2005; Altaf et al. 2014), which strongly limits their ability to provide reliable forecasts during the critical period of the surge. The results of Fig. 8 confirm that both the hybrid covariance and, in particular, the OSA smoothing formulations significantly enhance the EnKF performances during those periods.

We further plot in Fig. 9 the hydrographs of the reference state and of the forecast and analysis states as resulting from all filters, at three stations close to the landfall areas. In general, one can see that the filters' forecasts consistently underestimate the true data, mainly just before and during the surge. This is due to the dissipative nature of the coarse forecast model, and to the coarse wind forcing. The analysis steps successfully bring the model closer to the truth, providing more accurate estimates over most of the assimilation period. Overall, ETKF-OSA, ETKF $F_{\mathrm{Hyb}}$, and $\mathrm{ETKF}_{\mathrm{Hyb}}-\mathrm{OSA}$ are able to provide viable improvements to the ETKF forecasts and analysis, particularly during the landfall period.

Figures 10 and 11 report the forecast errors of water elevations at respectively 0600 UTC 13 September 2008 (i.e., one hour before the hurricane landfall) and 0800 UTC 13 September 2008 (i.e., one hour after the landfall). Compared to the ETKF, the ETKF-OSA and ETKF $_{\text {Hyb }}$ provide more reliable forecasts, especially near the coastline. This is more pronounced with the ETKF $_{\mathrm{Hyb}}-\mathrm{OSA}$ where the forecast errors are clearly reduced compared to all the other filters.

We finally investigate the sensitivity of the filters performances to the ensemble size. More specifically, we rerun all the experiments using twice and half the ensembles (used in Fig. 6) and report the average RMSEs of the maximum water level forecasts in Figs. 12 and 13, respectively. Increasing the ensemble size improves the performances of all the filters, but only slightly (roughly $5 \%$ ) relatively to the increase (almost double) in the computational cost. The background error covariances 

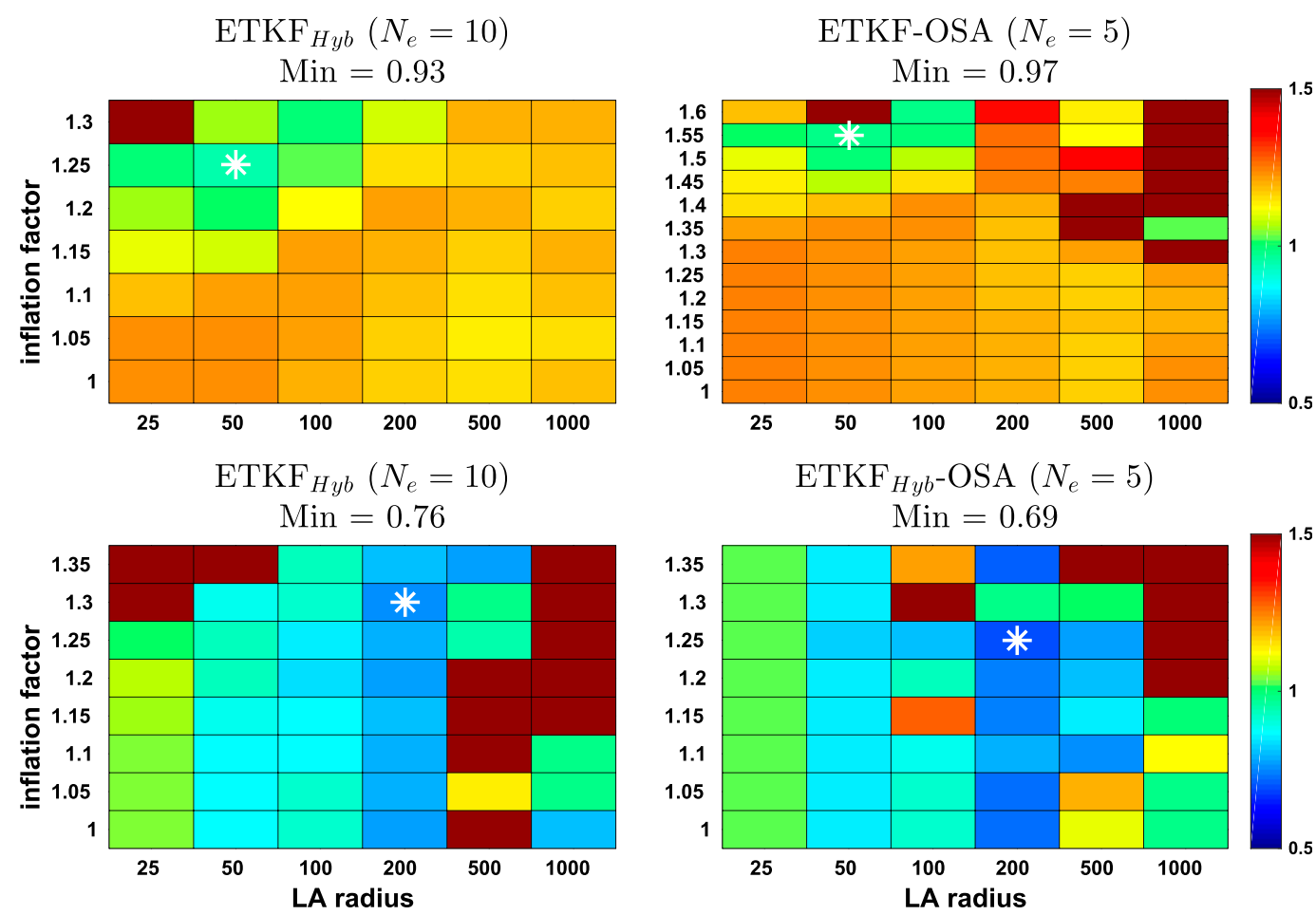

FIG. 13. As in Fig. 6, but using 10 members with ETKF and ETKF $_{\mathrm{Hyb}}$, and 5 members with ETKF-OSA and $\mathrm{ETKF}_{\mathrm{Hyb}}-\mathrm{OSA}$.

and the associated estimates are more efficiently estimated using the hybrid and OSA smoothing techniques than by doubling the ensemble size. For instance, the minimum RMSE achieved by the ETKF with 40 members is larger than that of the $\mathrm{ETKF}_{\mathrm{Hyb}}$ with 20 members and those of ETKF-OSA and ETKF $\mathrm{Hyb}_{\mathrm{H}}$-OSA with 10 members. Reducing the ensemble size, on the other hand, slightly deteriorates the forecasting skills of all the filters (by less than 10\%), except for the ETKF-OSA, whose minimum RMSE increases from 0.68 using 10 members to 0.97 with 5 members, corresponding to a deterioration of around $30 \%$. An ensemble of 5 members is obviously very small for the studied assimilation problem. Performing the two-stage update, based on such a small ensemble introduces more undersampling errors during both smoothing and analysis steps, thereby leading to deteriorated estimates. In this case, the ETKF with a reasonable ensemble of 10 members is shown to be more efficient than ETKF-OSA with only 5 members. With an ensemble of 10 members, however, the two-stage update step becomes more efficient and ETKF-OSA outperforms ETKF. Hybridizing the (cross) covariances, $\mathrm{ETKF}_{\mathrm{Hyb}} \mathrm{OSA}$, with only 5 members, results in a relative improvement of around $29 \%$ compared to the ETKF-OSA with the same ensemble size. This supports our combined hybrid-OSA approach, suggesting that the hybrid formulation could be used as an efficient strategy to allow for the implementation of the OSA smoothing formulation with small ensembles.

Figures 14 and 15 plot the spatial distribution of the maximum error over the coastal domain as resulting from the ETKF and $\mathrm{ETKF}_{\mathrm{Hyb}}$ with 40 and 10 members, respectively, and from the OSA smoothing schemes with 20 and 5 members, respectively. From Fig. 14, one can notice similar results as in Fig. 7, with a reasonable improvement in the filters' capabilities in estimating the maximum surge. A similar behavior can also be seen from Fig. 15 where the filters performances slightly degrade compared to those of Fig. 7, except for the ETKF-OSA where the degradation in the filter performance becomes more pronounced. The results also suggest that the filters' performances are more dependent on the filtering schemes than on the ensemble size, which further supports our approach. The results further suggest that the temporal variations of the dynamics inside the bay are not pronounced so that they could be well captured with a representative static ensemble complemented with a small dynamic one.

\section{Conclusions}

We introduced and successfully tested a new data assimilation system for efficient storm surge forecasting 

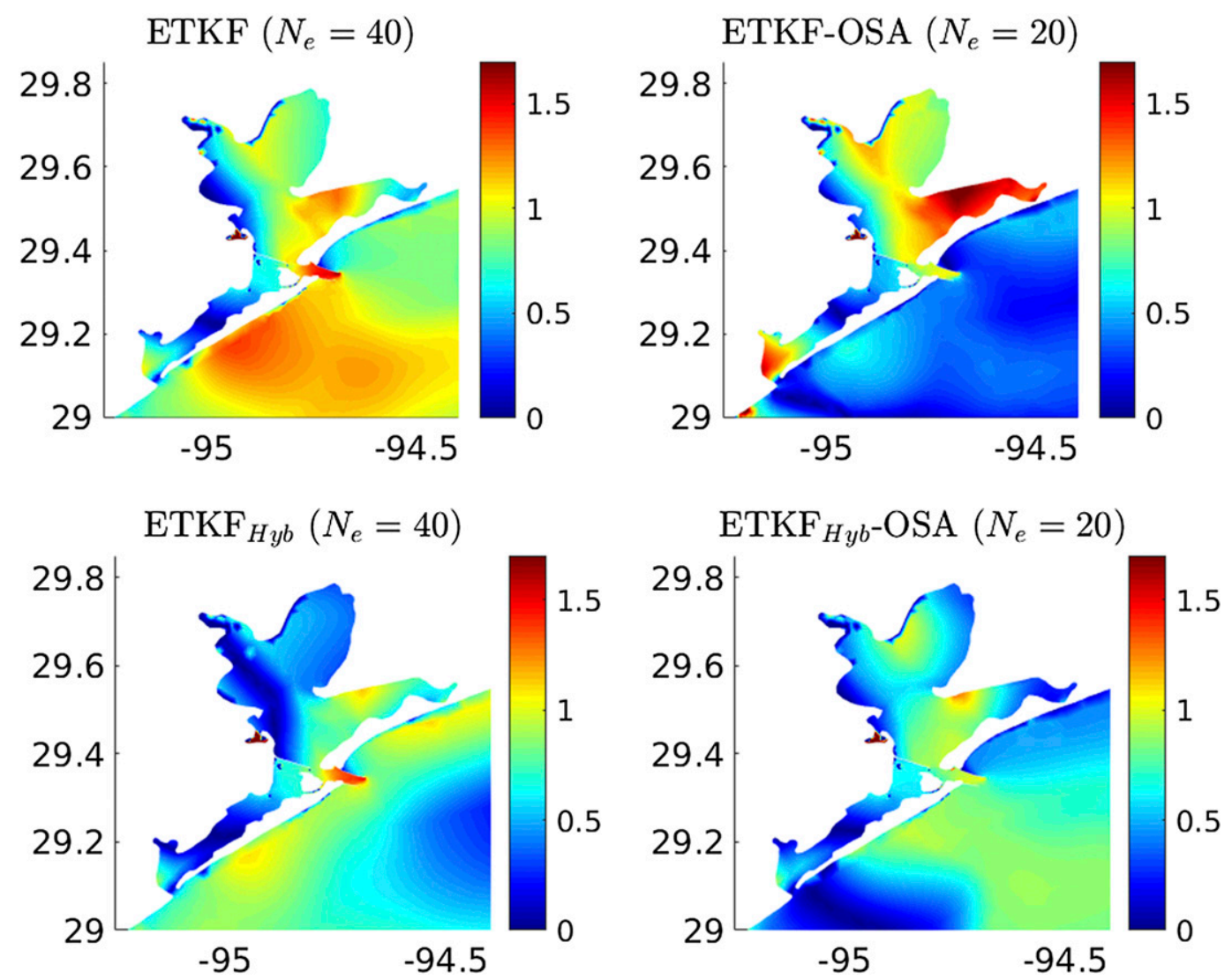

FIG. 14. As in Fig. 7, but the results are presented for the best configurations of ETKF and ETKF $_{\mathrm{Hyb}}$ with 40 members and ETKF-OSA and ETKF $\mathrm{Hyb}$-OSA with 20 members.

with the ensemble Kalman filter by combining two boosting auxiliary techniques, the hybrid covariance and the one-step-ahead smoothing formulations in the context of the ETKF. We further evaluated the filter's performances and compared its results to that of the standard ETKF for real-time short-range forecasting of the surge associated with Hurricane Ike that swirled over the Gulf of Mexico in September 2008.

OSA smoothing introduces a smoothing step of the previous analysis with the future observation before applying another round of forecasting step. This was shown to improve the background ensemble statistics and thus the filter's estimates at the cost of doubling the computational cost. The hybrid covariance technique also helps mitigating for the deficiency of the filter error covariance matrix by complementing the flow-dependent EnKF error covariance with a (preselected) static covariance. It was found to be particularly beneficial when the filter is implemented with small ensembles. The idea behind combining these two techniques for storm surge forecasting is to exploit the OSA smoothing step for improving the standard EnKF behavior during the strongly nonlinear storm surge periods while allowing for an efficient implementation of the filter with small ensembles to reduce the computational load using the hybrid formulation.

Observing system simulation experiments (OSSEs) were conducted in which the reference states were generated using a validated high-resolution ADCIRC run of the western North Atlantic and Gulf of Mexico forced with high-fidelity wind fields. A coarser ADCIRC configuration of the Gulf of Mexico forced with coarse global wind (generated by the dynamic Holland wind model) was used as the forecast model. The assimilation results demonstrated the efficiency of the filtering approach, producing promising results in terms of significantly improving the storm surge forecasts without noticeable increase in the computational cost. In particular, the filter enhanced the forecasting skill of the maximum water elevation over the landfall area and decreased the water elevation errors in the hours preceding the landfall compared to the standard ETKF. Appropriate tuning of inflation and localization parameters was necessary to achieve reliable performances, with the proposed filter being also more robust to the 

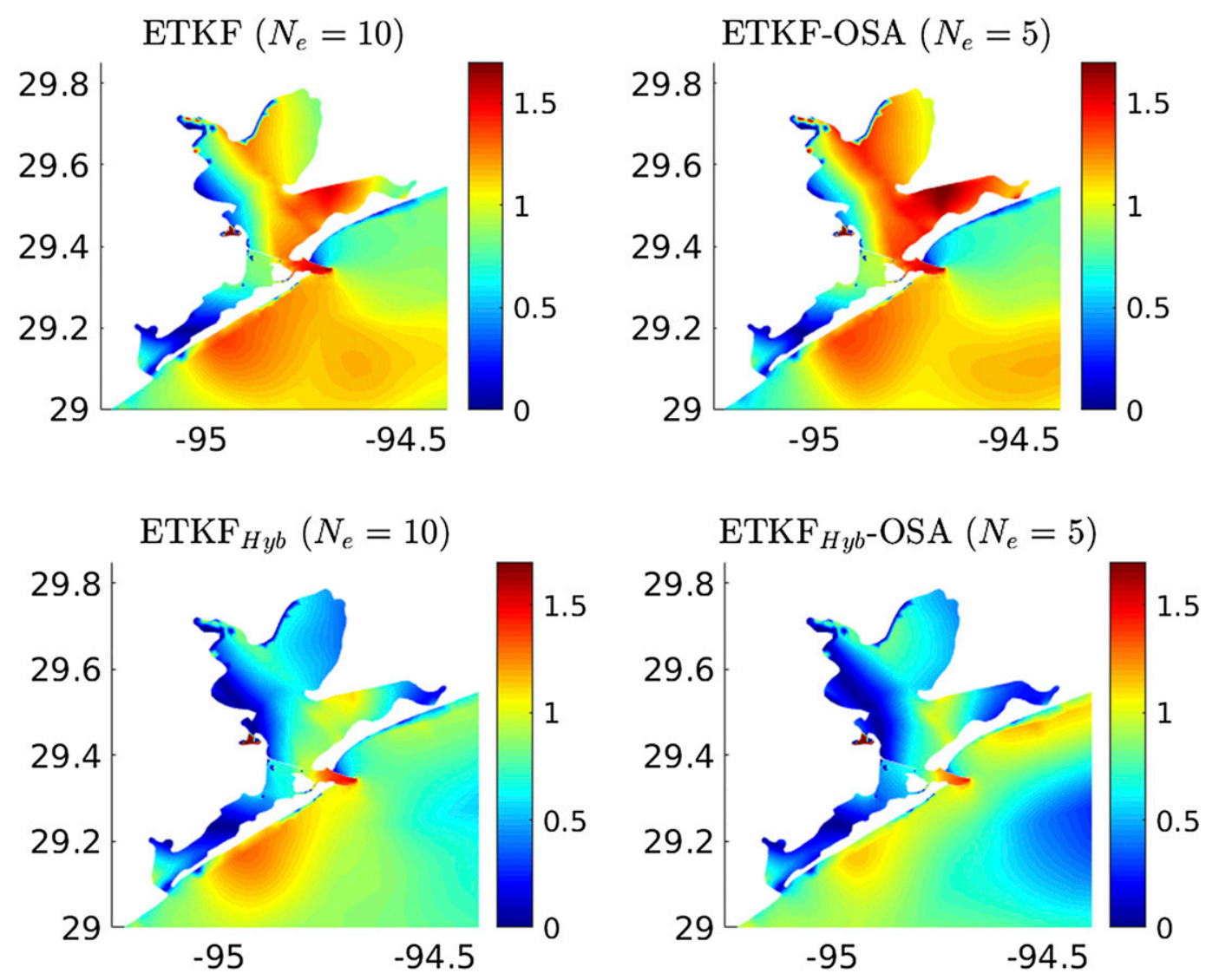

FIG. 15. As in Fig. 7, but the results are presented for the best configurations of ETKF and ETKF $_{\mathrm{Hyb}}$ with 10 members and ETKF-OSA and ETKF $_{\mathrm{Hyb}^{-}} \mathrm{OSA}$ with 5 members.

choice of these parameters. The OSA smoothing schemes required larger inflation to preserve enough ensemble spread after their two forecasting steps.

Overall, our results clearly suggest that the proposed filtering scheme provides a promising approach for efficient storm surge forecasting at reasonable computational cost. One may also consider using the so-called ensemble dressing forecast technique (Mel and Lionello 2014b; Mel et al. 2014), which try to infer information about the forecast uncertainty directly from the uncertainty in the meteorological forcing fields, and possibly from the prior uncertainties of the ensemble prediction system itself (Mel and Lionello 2016), as a way to reduce the number of model runs during the filter forecast step. Finally, in this study, the weights of the stationary covariances and cross covariances in the hybrid schemes were tuned based on trial-and-error experiments. It will be useful to conduct a more extensive set of experiments in future studies to fully investigate the filters' sensitivity to the values of these parameters, and also to develop efficient adaptive schemes for online tuning of their values.
Acknowledgments. This work is supported by King Abdullah University of Science and Technology Award CRG3-2156. The research made use of the Shaheen supercomputer resources.

\section{REFERENCES}

Ait-El-Fquih, B., and I. Hoteit, 2016: A variational Bayesian multiple particle filtering scheme for large-dimensional systems. IEEE Trans. Signal. Process., 64, 5409-5422, https:// doi.org/10.1109/TSP.2016.2580524.

_- M. El Gharamti, and I. Hoteit, 2016: A Bayesian consistent dual ensemble Kalman filter for state-parameter estimation in subsurface hydrology. Hydrol. Earth Syst. Sci., 20, 3289-3307, https://doi.org/10.5194/hess-20-3289-2016.

Altaf, M., T. Butler, X. Luo, C. Dawson, T. Mayo, and I. Hoteit, 2013: Improving short-range ensemble Kalman storm surge forecasting using robust adaptive inflation. Mon. Wea. Rev., 141, 2705-2720, https://doi.org/10.1175/MWR-D-12-00310.1.

- - , T. Mayo, X. Luo, C. Dawson, A. Heemink, and I. Hoteit, 2014: A comparison of ensemble Kalman filters for storm surge assimilation. Mon. Wea. Rev., 142, 2899-2914, https://doi.org/10.1175/MWR-D-13-00266.1.

Anderson, J. L., 2001: An ensemble adjustment Kalman filter for data assimilation. Mon. Wea. Rev., 129, 2884-2903, 
https://doi.org/10.1175/1520-0493(2001)129<2884:AEAKFF $>$ 2.0.CO;2.

Bennett, A., 1992: Inverse Methods in Physical Oceanography. Cambridge University Press, 364 pp.

Berg, R., 2009: Tropical cyclone report: Hurricane Ike (114 September 2008). NHC Rep. AL092008, National Hurricane Center, 55 pp., https://www.hcfcd.org/media/1240/ nhc_hurricane_ike_report.pdf.

Beven, K., 1989: Changing ideas in hydrology-The case of physically-based models. J. Hydrol., 105, 157-172, https:// doi.org/10.1016/0022-1694(89)90101-7.

Bishop, C. H., B. J. Etherton, and S. J. Majumdar, 2001: Adaptive sampling with ensemble transform Kalman filter. Part I: Theoretical aspects. Mon. Wea. Rev., 129, 420-436, https://doi.org/ 10.1175/1520-0493(2001)129<0420:ASWTET>2.0.CO;2.

Blake, E. S., E. N. Rappaport, and C. W. Landsea, 2007: The deadliest, costliest, and most intense United States tropical cyclones from 1851 to 2006 (and other frequently requested hurricane facts). NOAA Tech. Memo. NWS TPC-5, NOAA/National Weather Service, National Centers for Environmental Prediction, National Hurricane Center, 45 pp.

Buizza, R., and T. N. Palmer, 1995: The singular-vector structure of the atmospheric global circulation. J. Atmos. Sci., 52, 1434 1456, https://doi.org/10.1175/1520-0469(1995)052<1434:TSVSOT> 2.0.CO;2.

_- M. Milleer, and T. N. Palmer, 1999: Stochastic representation of model uncertainties in the ECMWF ensemble prediction system. Quart. J. Roy. Meteor. Soc., 125, 2887-2908, https:// doi.org/10.1002/qj.49712556006.

Burgers, G., P. J. van Leeuwen, and G. Evensen, 1998: On the analysis scheme in the ensemble Kalman filter. Mon. Wea. Rev., 126, 1719-1724, https://doi.org/10.1175/1520-0493(1998) 126<1719:ASITEK > 2.0.CO;2.

Butler, T., M. Altaf, C. Dawson, I. Hoteit, X. Luo, and T. Mayo, 2012: Data assimilation within the Advanced Circulation (ADCIRC) modeling framework for hurricane storm surge forecasting. Mon. Wea. Rev., 140, 2215-2231, https://doi.org/ 10.1175/MWR-D-11-00118.1.

Chassignet, E. P., A. Pascual, J. Tintoré, and J. Verron, Eds., 2018: New Frontiers in Operational Oceanography. CreateSpace Independent Publishing Platform, $812 \mathrm{pp}$.

Dawson, C., J. J. Westerink, J. C. Feyen, and D. Pothina, 2006: Continuous, discontinuous and coupled discontinuouscontinuous Galerkin finite element methods for the shallow water equations. Int. J. Numer. Methods Fluids, 52, 63-88, https://doi.org/10.1002/fld.1156.

Desbouvries, F., Y. Petetin, and B. Ait-El-Fquih, 2011: Direct, prediction- and smoothing-based Kalman and particle filter algorithms. Signal Process., 91, 2064-2077, https://doi.org/10.1016/ j.sigpro.2011.03.013

Dietrich, J., and Coauthors, 2011a: Hurricane Gustav (2008) waves and storm surge: Hindcast, synoptic analysis, and validation in southern Louisiana. Mon. Wea. Rev., 139, 2488-2522, https:// doi.org/10.1175/2011MWR3611.1.

__ , and Coauthors, 2011b: Modeling hurricane waves and storm surge using integrally-coupled, scalable computations. Coastal Eng., 58, 45-65, https://doi.org/10.1016/j.coastaleng.2010.08.001.

Evensen, G., 2003: The ensemble Kalman filter: Theoretical formulation and practical implementation. Ocean Dyn., 53, 343-367, https://doi.org/10.1007/s10236-003-0036-9.

Fleming, J. G., C. W. Fulcher, R. A. Luettich, B. D. Estrade, G. D. Allen, and H. S. Winer, 2008: A real time storm surge forecasting system using ADCIRC. Proc. 10th Int. Conf. on
Estuarine and Coastal Modeling, Newport, RI, American Society of Civil Engineers, 893-912, https://doi.org/10.1061/ 40990(324)48.

Flowerdew, J., K. Horsburgh, C. Wilson, and K. Mylne, 2010: Development and evaluation of an ensemble forecasting system for coastal storm surges. Quart. J. Roy. Meteor. Soc., 136, 1444-1456, https://doi.org/10.1002/qj.648.

_, K. Mylne, C. Jones, and H. Titley, 2013: Extending the forecast range of the UK storm surge ensemble. Quart. J. Roy. Meteor. Soc., 139, 184-197, https://doi.org/10.1002/qj.1950.

Ghil, M., 1989: Meteorological data assimilation for oceanographers. Part I: Description and theoretical framework. Dyn. Atmos. Oceans, 13, 171-218, https://doi.org/10.1016/03770265(89)90040-7.

Hamill, T. M., and C. Snyder, 2000: A hybrid ensemble Kalman filter-3D variational analysis scheme. Mon. Wea. Rev., 128, 2905-2919, https://doi.org/10.1175/1520-0493(2000)128<2905: AHEKFV $>2.0 . \mathrm{CO} ; 2$.

Heaps, N., 1983: Storm surges, 1967-1982. Geophys. J. Int., 74, 331376, https://doi.org/10.1111/j.1365-246X.1983.tb01883.x.

Holland, G. J., 1980: An analytic model of the wind and pressure profiles in hurricanes. Mon. Wea. Rev., 108, 1212-1218, https://doi.org/10.1175/1520-0493(1980)108<1212:AAMOTW > 2.0.CO;2

Hope, M. E., and Coauthors, 2013: Hindcast and validation of Hurricane Ike (2008) waves, forerunner, and storm surge. J. Geophys. Res. Oceans, 118, 4424-4460, https://doi.org/ 10.1002/jgrc.20314.

Hoteit, I., D. T. Pham, and J. Blum, 2002: A simplified reduced order Kalman filtering and application to altimetric data assimilation in Tropical Pacific. J. Mar. Syst., 36, 101-127, https:// doi.org/10.1016/S0924-7963(02)00129-X.

— , G. Korres, and G. Triantafyllou, 2005: Comparison of extended and ensemble based Kalman filters with low and high-resolution primitive equations ocean models. Nonlinear Processes Geophys., 12, 755-765, https://doi.org/10.5194/npg12-755-2005.

— X. Luo, and D. T. Pham, 2012: Particle Kalman filtering: A nonlinear Bayesian framework for ensemble Kalman filters. Mon. Wea. Rev., 140, 528-542, https://doi.org/10.1175/ 2011MWR3640.1.

, T. Hoar, G. Gopalakrishnan, N. Collins, J. Anderson, B. Cornuelle, A. Köhl, and P. Heimbach, 2013: A MITGCM/DART ensemble analysis and prediction system with application to the Gulf of Mexico. Dyn. Atmos. Oceans, 63, 1-23, https:// doi.org/10.1016/j.dynatmoce.2013.03.002.

—, D.-T. Pham, M. Gharamti, and X. Luo, 2015: Mitigating observation perturbation sampling errors in the stochastic EnKF. Mon. Wea. Rev., 143, 2918-2936, https://doi.org/ 10.1175/MWR-D-14-00088.1.

Houtekamer, P. L., and H. L. Mitchell, 1998: Data assimilation using an ensemble Kalman filter technique. Mon. Wea. Rev., 126, 796-811, https://doi.org/10.1175/1520-0493(1998)126<0796: DAUAEK $>2.0 . \mathrm{CO} ; 2$.

Kalman, R., 1960: A new approach to linear filtering and prediction problems. J. Basic Eng., 82, 35-45, https://doi.org/10.1115/ 1.3662552 .

Kalnay, E., and S.-C. Yang, 2010: Accelerating the spin-up of ensemble Kalman filtering. Quart. J. Roy. Meteor. Soc., 136, 1644-1651, https://doi.org/10.1002/qj.652.

Kennedy, A. B., and Coauthors, 2011: Origin of the Hurricane Ike forerunner surge. Geophys. Res. Lett., 38, L08608, https:// doi.org/10.1029/2011GL047090. 
Liu, B., B. Ait-El-Fquih, and I. Hoteit, 2016: Efficient kernel-based ensemble Gaussian mixture filtering. Mon. Wea. Rev., 144, 781-800, https://doi.org/10.1175/MWR-D-14-00292.1.

Lorenc, A. C., 2003: The potential of the ensemble Kalman filter for NWP-A comparison with 4D-Var. Quart. J. Roy. Meteor. Soc., 129, 3183-3203, https://doi.org/10.1256/qj.02.132.

Luettich, R. A., and J. J. Westerink, 2004: Formulation and numerical implementation of the 2D/3D ADCIRC finite element model version 44. XX. R. Luettich, $74 \mathrm{pp}$.

$\longrightarrow$, and - 2007: A parallel ADvanced CIRCulation model for oceanic coastal and estuarine waters. Tech. Rep., ADCIRC, http://www.adcirc.org.

Luo, X., and I. Hoteit, 2011: Robust ensemble filtering and its relation to covariance inflation in the ensemble Kalman filter. Mon. Wea. Rev., 139, 3938-3953, https://doi.org/10.1175/ MWR-D-10-05068.1.

Lynch, D. R., and W. G. Gray, 1979: A wave equation model for finite element tidal computations. Comput. Fluids, 7, 207-228, https://doi.org/10.1016/0045-7930(79)90037-9.

Malanotte-Rizzoli, P., R. E. Young, and D. B. Haidvogel, 1989: Initialization and data assimilation experiments with a primitive equation model. Dyn. Atmos. Oceans, 13, 349-378, https:// doi.org/10.1016/0377-0265(89)90046-8.

McMillan, H. K., and J. Brasington, 2008: End-to-end flood risk assessment: A coupled model cascade with uncertainty estimation. Water Resour. Res., 44, W03419, https://doi.org/ 10.1029/2007WR005995.

McRobie, A., T. Spencer, and H. Gerritsen, 2005: The big flood: North Sea storm surge. Philos. Trans. Roy. Soc. London, 363, 1263-1270, https://doi.org/10.1098/rsta.2005.1567.

Mel, R., and P. Lionello, 2014a: Storm surge ensemble prediction for the city of Venice. Wea. Forecasting, 29, 1044-1057, https:// doi.org/10.1175/WAF-D-13-00117.1.

$\longrightarrow$, and — 2014b: Verification of an ensemble prediction system for storm surge forecast in the Adriatic Sea. Ocean Dyn., 64, 1803-1814, https://doi.org/10.1007/s10236-014-0782-x.

$\longrightarrow$, and - 2016: Probabilistic dressing of a storm surge prediction in the Adriatic Sea. Adv. Meteor., 2016, 3764519, https://doi.org/10.1155/2016/3764519.

—, D. P. Viero, L. Carniello, A. Defina, and L. D'Alpaos, 2014: Simplified methods for real-time prediction of storm surge uncertainty: The city of Venice case study. Adv. Water Resour., 71, 177-185, https://doi.org/10.1016/j.advwatres.2014.06.014.

Murty, T. S., R. A. Flather, and R. Henry, 1986: The storm surge problem in the Bay of Bengal. Prog. Oceanogr., 16, 195-233, https://doi.org/10.1016/0079-6611(86)90039-X.

Pham, D. T., 2001: Stochastic methods for sequential data assimilation in strongly nonlinear systems. Mon. Wea. Rev., 129, 1194-1207, https://doi.org/10.1175/1520-0493(2001)129<1194: SMFSDA $>2.0 . \mathrm{CO} ; 2$.

, J. Verron, and M. C. Roubaud, 1998: A singular evolutive extended Kalman filter for data assimilation in oceanography. J. Mar. Syst., 16, 323-340, https://doi.org/10.1016/S09247963(97)00109-7.
Raboudi, N. F., B. Ait-El-Fquih, and I. Hoteit, 2018: Ensemble Kalman filtering with one-step-ahead smoothing. Mon. Wea. Rev., 146, 561-581, https://doi.org/10.1175/MWR-D-17-0175.1.

Sakov, P., and L. Bertino, 2011: Relation between two common localisation methods for the EnKF. Comput. Geosci., 15, 225237, https://doi.org/10.1007/s10596-010-9202-6.

Siripatana, A., T. Mayo, I. Sraj, O. Knio, C. Dawson, O. Le Maitre, and I. Hoteit, 2017: Assessing an ensemble Kalman filter inference of Manning's $n$ coefficient of an idealized tidal inlet against a polynomial chaos-based MCMC. Ocean Dyn., 67, 1067-1094, https://doi.org/10.1007/s10236-017-1074-z.

Song, H., I. Hoteit, B. D. Cornuelle, and A. C. Subramanian, 2010: An adaptive approach to mitigate background covariance limitations in the ensemble Kalman filter. Mon. Wea. Rev., 138, 2825-2845, https://doi.org/10.1175/2010MWR2871.1.

Tanaka, S., S. Bunya, J. J. Westerink, C. Dawson, and R. A. Luettich, 2011: Scalability of an unstructured grid continuous Galerkin based hurricane storm surge model. J. Sci. Comput., 46, 329-358, https://doi.org/10.1007/s10915-010-9402-1.

Tippett, M. K., J. L. Anderson, C. H. Bishop, T. M. Hamill, and J. S. Whitaker, 2003: Ensemble square root filters. Mon. Wea. Rev., 131, 1485-1490, https://doi.org/10.1175/1520-0493(2003) $131<1485:$ ESRF $>2.0 . C O ; 2$.

Torres, J. M., B. Bass, J. N. Irza, J. Proft, A. Sebastian, C. Dawson, and P. Bedient, 2017: Modeling the hydrodynamic performance of a conceptual storm surge barrier system for the Galveston Bay region. J. Waterway Port Coastal Ocean Eng., 143, https://doi.org/10.1061/(ASCE)WW.1943-5460.0000389.

Toye, H., P. Zhan, G. Gopalakrishnan, A. R. Kartadikaria, H. Huang, O. Knio, and I. Hoteit, 2017: Ensemble data assimilation in the Red Sea: Sensitivity to ensemble selection and atmospheric forcing. Ocean Dyn., 67, 915-933, https:// doi.org/10.1007/s10236-017-1064-1.

Tsiaras, K. P., I. Hoteit, S. Kalaroni, G. Petihakis, and G. Triantafyllou, 2017: A hybrid ensemble-OI Kalman filter for efficient data assimilation into a 3-D biogeochemical model of the Mediterranean. Ocean Dyn., 67, 673-690, https:// doi.org/10.1007/s10236-017-1050-7.

Wang, X., and C. H. Bishop, 2003: A comparison of breeding and ensemble transform Kalman filter ensemble forecast schemes. J. Atmos. Sci., 60, 1140-1158, https://doi.org/10.1175/15200469(2003)060<1140:ACOBAE > 2.0.CO;2.

,-- , and S. J. Julier, 2004: Which is better, an ensemble of positive-negative pairs or a centered spherical simplex ensemble? Mon. Wea. Rev., 132, 1590-1605, https://doi.org/ 10.1175/1520-0493(2004)132<1590:WIBAEO > 2.0.CO;2.

_ T. M. Hamill, J. S. Whitaker, and C. H. Bishop, 2009: A comparison of the hybrid and EnSRF analysis schemes in the presence of model errors due to unresolved scales. Mon. Wea. Rev., 137, 3219-3232, https://doi.org/10.1175/2009MWR2923.1.

Zhong, L., M. Li, and D.-L. Zhang, 2010: How do uncertainties in hurricane model forecasts affect storm surge predictions in a semi-enclosed bay? Estuarine Coastal Shelf Sci., 90, 61-72, https://doi.org/10.1016/j.ecss.2010.07.001. 